\title{
UICN
}

\section{Estado Verde de las Especies de la UICN}

Un estándar mundial para medir la recuperación de especies y evaluar el impacto de la conservación

Preparado por el Grupo de Tareas de la CSE UICN para la Conservación Eficaz de las Especies Versión 2.0 


\section{Sobre UICN}

La UICN es una unión de miembros compuesta tanto por gobiernos como por organizaciones de la sociedad civil. Brinda a organizaciones públicas, privadas y no gubernamentales, el conocimiento y las herramientas que permiten que el progreso humano, el desarrollo económico y la conservación de la naturaleza se desarrollen juntos.

Creada en 1948, la UICN es ahora la red ambiental más grande y diversa, aprovechando el conocimiento, los recursos y el alcance de más de 1.400 organizaciones miembros y unos 18.000 expertos. Es un proveedor líder de datos de conservación, evaluaciones y análisis. Su amplia membresía permite a la UICN desempeñar el papel de incubadora y repositorio confiable de mejores prácticas, herramientas y estándares internacionales.

La UICN proporciona un espacio neutral en el que diversas partes interesadas, incluidos gobiernos, ONG, científicos, empresas, comunidades locales, organizaciones de pueblos indígenas y otras, puedan trabajar juntas para forjar e implementar soluciones a los desafíos ambientales y lograr un desarrollo sostenible.

Al trabajar con muchos socios y seguidores, la UICN implementa una amplia y diversa cartera de proyectos de conservación en todo el mundo. Combinando la ciencia más reciente con el conocimiento tradicional de las comunidades locales, estos proyectos trabajan para revertir la pérdida de hábitat, restaurar los ecosistemas y mejorar el bienestar de las personas.

www.iucn.org/es

https://twitter.com/IUCN/ 


\section{Estado Verde de las Especies de la UICN}

Un estándar mundial para medir la recuperación de especies y evaluar el impacto de la conservación

Preparado por el Grupo de Tareas de la CSE UICN para la Conservación Eficaz de las Especies Versión 2.0 
La designación de entidades geográficas y la presentación del material en esta publicación no implican la expresión de ninguna opinión por parte de la UICN ni de ninguna otra organización participante respecto a la condición jurídica de ningún país, territorio o área, o de sus autoridades, o referente a la delimitación de sus fronteras y límites.

Las opiniones expresadas en esta publicación no reflejan necesariamente las de la UICN ni de otras organizaciones participantes.

Preparado por el Grupo de Tareas de la CSE UICN para la Conservación Eficaz de las Especies. Aprobado en la $102^{\circ}$ Reunión del Consejo de la UICN Gland, Suiza (01/12/2020).

La UICN se complace en agradecer el apoyo de sus socios marco por su financiación del programa de la UICN: el Ministerio de Asuntos Exteriores de Dinamarca; el Ministerio de Asuntos Exteriores de Finlandia; el Gobierno de Francia y la Agencia Francesa de Desarrollo (AFD); el Ministerio del Medio Ambiente de la República de Corea; la Agencia Noruega para la Cooperación al Desarrollo (Norad); el Agencia Sueca de Cooperación Internacional para el Desarrollo (Asdi); la Agencia Suiza para el Desarrollo y la Cooperación (COSUDE) y el Departamento de Estado de Estados Unidos.

Esta publicación ha sido posible, en parte, gracias a la generosidad del Consejo de Investigaciones sobre el Medio Ambiente Natural (NERC) del Reino Unido, WWF US, Re:wild (antes GWC), la Comisión para la Supervivencia de las Especies de la UICN, la Universidad de Oxford, la Universidad de Stony Brook y la Fundación Príncipe Alberto (a través de la Iniciativa de Conservación de Cambridge).

La UICN o demás organizaciones participantes no reivindican ninguna responsabilidad por los errores $u$ omisiones que puedan ocurrir en la traducción a otros idiomas de este documento, cuya versión original es el idioma original. En caso de discrepancia, remítase, por favor, a la edición original. Título de la edición original: IUCN Green Status of Species: A global standard for measuring species recovery and assessing conservation impact. Version 2.0. Publicado por: UICN, Gland, Suiza. DOI: https://doi.org/10.2305/IUCN.CH.2021.02.en

Publicado por: $\quad$ UICN, Gland, Suiza

Producido por: El Grupo de Tareas de la CSE UICN para la Conservación Eficaz de las Especies

Derechos $\quad$ C 2021 UICN, Unión Internacional para la Conservación de la Naturaleza y de los Recursos Naturales

reservados: Se autoriza la reproducción de esta publicación con fines educativos y otros fines no comerciales sin permiso escrito previo de parte de quien detenta los derechos de autor con tal de que se mencione la fuente.

Se prohíbe reproducir esta publicación para la venta o para otros fines comerciales sin permiso escrito previo de quien detenta los derechos de autor.

Citación UICN (2021). Estado Verde de las Especies de la UICN: Un estándar mundial para medir la recuperación de especies y evaluar el recomendada: impacto de la conservación. Versión 2.0. Gland, Suiza: UICN.

ISBN: $\quad$ 978-2-8317-2126-2 (PDF)

DOI: https://doi.org/10.2305/IUCN.CH.2021.02.es

Fotografía [portada] Rana gladiadora gigante, Hypsiboas boans, en la Reserva Natural de Cocobolo, Panamá @ Robin Moore

de la cubierta: [contraportada] Magnolia orbiculata en el Jardín Botánico Nacional de Cuba @ Ernesto Teste

Diseño: Jessica Avanidhar, jessavanidhar.com

Traducción: José Javier Torres Rodríguez 


\section{Îndice}

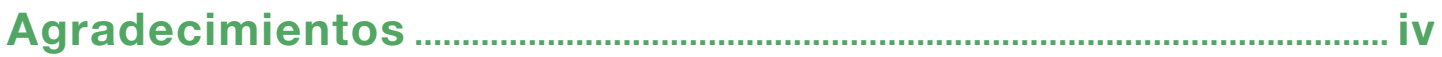

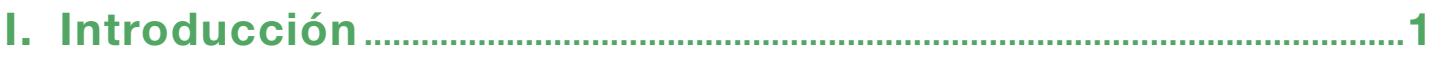

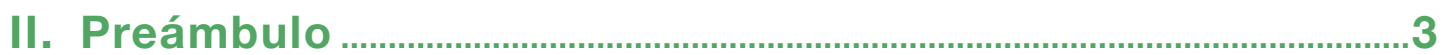

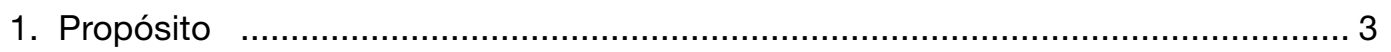

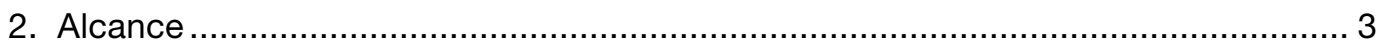

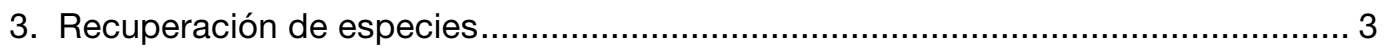

4. Relación con la Lista Roja de la UICN ............................................................... 4

5. Relación con la planificación y las prioridades en materia de conservación............. 4

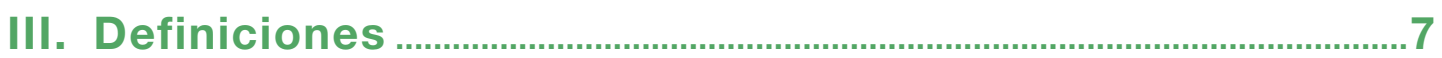

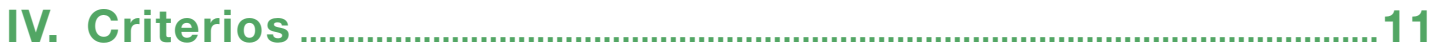

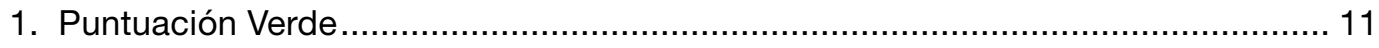

2. Indicadores de impacto de la conservación........................................................ 12

3. Categorías y umbrales ................................................................................. 13

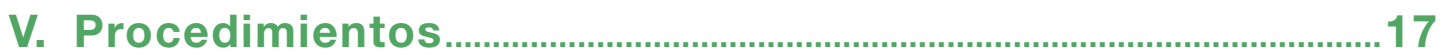

1. Determinación del área de distribución............................................................... 17

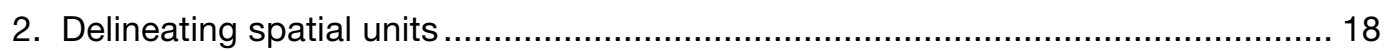

3. Evaluación del estado en una unidad espacial ................................................... 19

4. Elaboración del escenario Actual contrafactual .................................................... 20

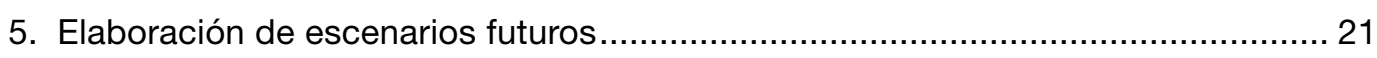

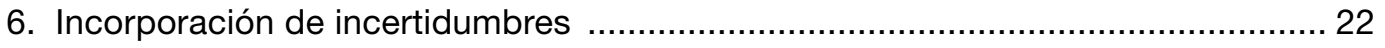

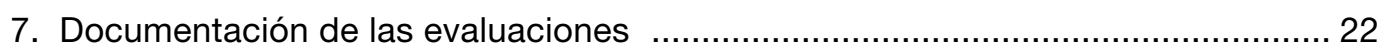

8. Comunicación de los resultados de la evaluación ................................................. 22

9. Evaluaciones regionales (incluidas las nacionales) .............................................. 22

VI. Referencias 


\section{Agradecimientos}

La UICN agradece la dedicación y los esfuerzos del Grupo de Trabajo para la Conservación Eficaz de las Especies (SCSTF, por sus siglas en inglés) al proponer los métodos del Estado Verde de las Especies de la UICN y al realizar pruebas rigurosas de los métodos propuestos mediante consultas, talleres y estudios científicos. El SCSTF fue copresidido por Barney Long y Elizabeth Bennett, y coordinado por Molly Grace. Los miembros del grupo central del SCSTF fueron: Resit Akçakaya, Craig Hilton-Taylor, Mike Hoffmann, E.J. Milner-Gulland, Richard Jenkins, Ana Nieto y Richard Young; otros miembros del SCSTF fueron: Thomas Brooks, Anna Heath, Simon Hedges, David Keith, David Mallon, Erik Meijaard, Ana Rodrigues, Jon Paul Rodríguez, PJ Stephenson y Simon Stuart. Debemos dar las gracias especialmente a Resit Akçakaya, que desempeñó un papel destacado en el desarrollo científico del método.

Para desarrollar el método del Estado Verde de las Especies, el SCSTF formó varios grupos de trabajo, que incluían tanto a miembros del SCSTF como a expertos externos: un Grupo de Trabajo de Objetivos y Puntos de Referencia, dirigido por PJ Stephenson; un Grupo de Trabajo sobre Escenarios, dirigido por E.J. Milner-Gulland; un Grupo de Trabajo sobre Viabilidad y Funcionalidad, dirigido por Resit Akçakaya; un Grupo de Trabajo sobre Unidades Espaciales, dirigido por Elizabeth Bennett y Molly Grace; un Grupo de Trabajo sobre Cuestiones Técnicas, dirigido por Craig Hilton-Taylor y Michael Hoffmann; y un Grupo de Trabajo sobre Comunicación, dirigido por Barney Long. Los miembros no pertenecientes al SCSTF que participaron en estos grupos fueron: Alison Boyer, Joe Bull, Onnie Byers, Cheli Cresswell Sinclair, Axel Hochkirch, Ackbar Joolia, Phil McGowan, Elaine Paterson, Eric Sanderson, Carrie Stengel, Ricardo Tejeda, Jessica Welch y el Grupo de Trabajo Técnico de la Lista Roja.

El SCSTF llevó a cabo una amplia prueba piloto del método del Estado Verde de las Especies con 181 especies, supervisada por Molly Grace. Más de 200 miembros del Grupo de Especialistas de la CSE y expertos independientes en especies ofrecieron su tiempo y conocimientos para llevar a cabo evaluaciones piloto de especies, que fueron fundamentales para perfeccionar el método. Quince de estas evaluaciones fueron patrocinadas por National Geographic y dirigidas por PJ Stephenson. Nigel Dudley y Hannah Timmins, de Equilibrium Research, dirigieron una consulta entre los posibles usuarios finales; los comentarios de los representantes de las empresas, la política y las ONG, entre otros, contribuyeron en gran medida a perfeccionar el método. Sarah Leonard llevó a cabo un proceso de consulta terminológica con las oficinas regionales de la UICN, los representantes regionales de la CSE de la UICN, zoológicos y acuarios, así como con periodistas, con el fin de facilitar la comunicación eficaz del producto final.

La labor del SCSTF ha sido posible gracias al generoso apoyo financiero del Consejo de Investigaciones del Medio Ambiente Natural (NERC) del Reino Unido, WWF US, Re:wild (antes GWC), la Comisión para la Supervivencia de las Especies de la UICN, la Universidad de Oxford, la Universidad de Stony Brook y la Fundación Príncipe Alberto (a través de la Iniciativa de Conservación de Cambridge). Agradecemos a las siguientes organizaciones su apoyo financiero a los talleres del SCSTF: el NERC, la Fundación Franklinia, la Fundación Príncipe Alberto (a través de la Iniciativa de Conservación de Cambridge) y el Fondo Semilla de Intercambio de Conocimientos de Oxford. Varias organizaciones han contribuido permitiendo a los miembros del SCSTF contribuir con su tiempo de trabajo: la Sociedad para la Conservación de la Vida Silvestre, la Sociedad Zoológica de Londres y Durrell.

Por último, la UICN está en deuda con los miembros de la Comisión y los representantes de las Organizaciones Miembro que participaron en los talleres de consulta o que presentaron observaciones y sugerencias durante el proceso de revisión de este Estándar. Estos aportes combinados han resultado en un sistema mucho más robusto, fácil de utilizar y de amplia aplicabilidad. 


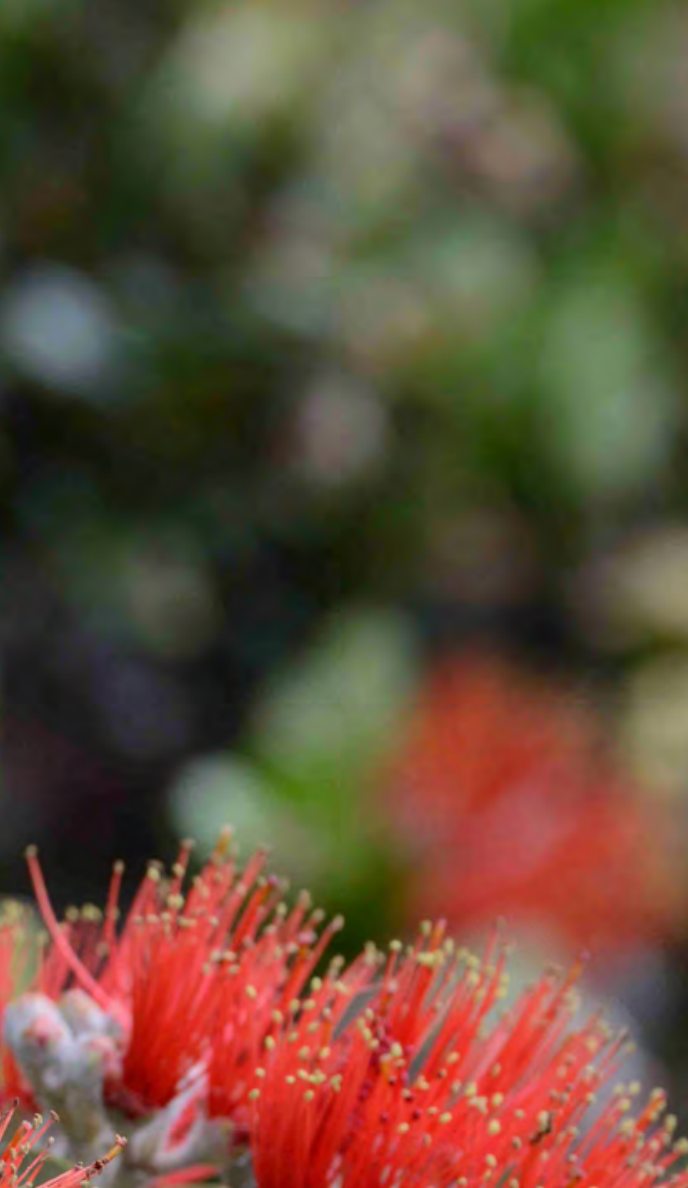

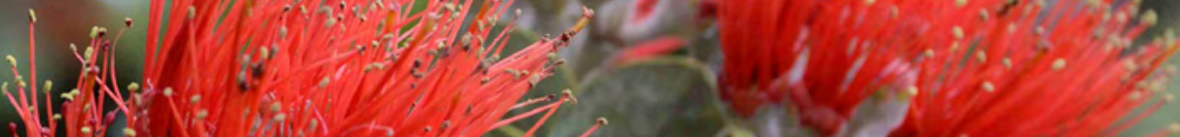

$\sqrt{1}+10$

- colst:

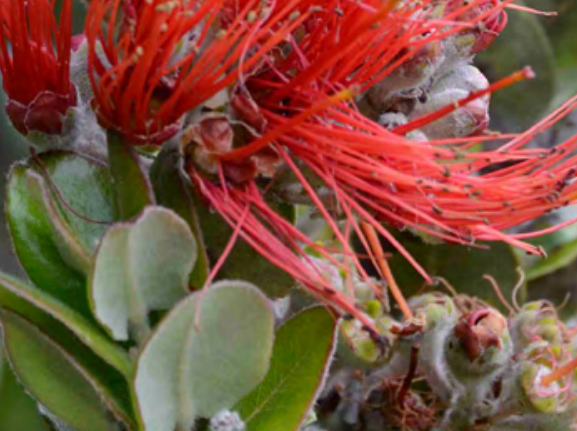

$2+\frac{1}{2}\left(\frac{1}{2}\right.$

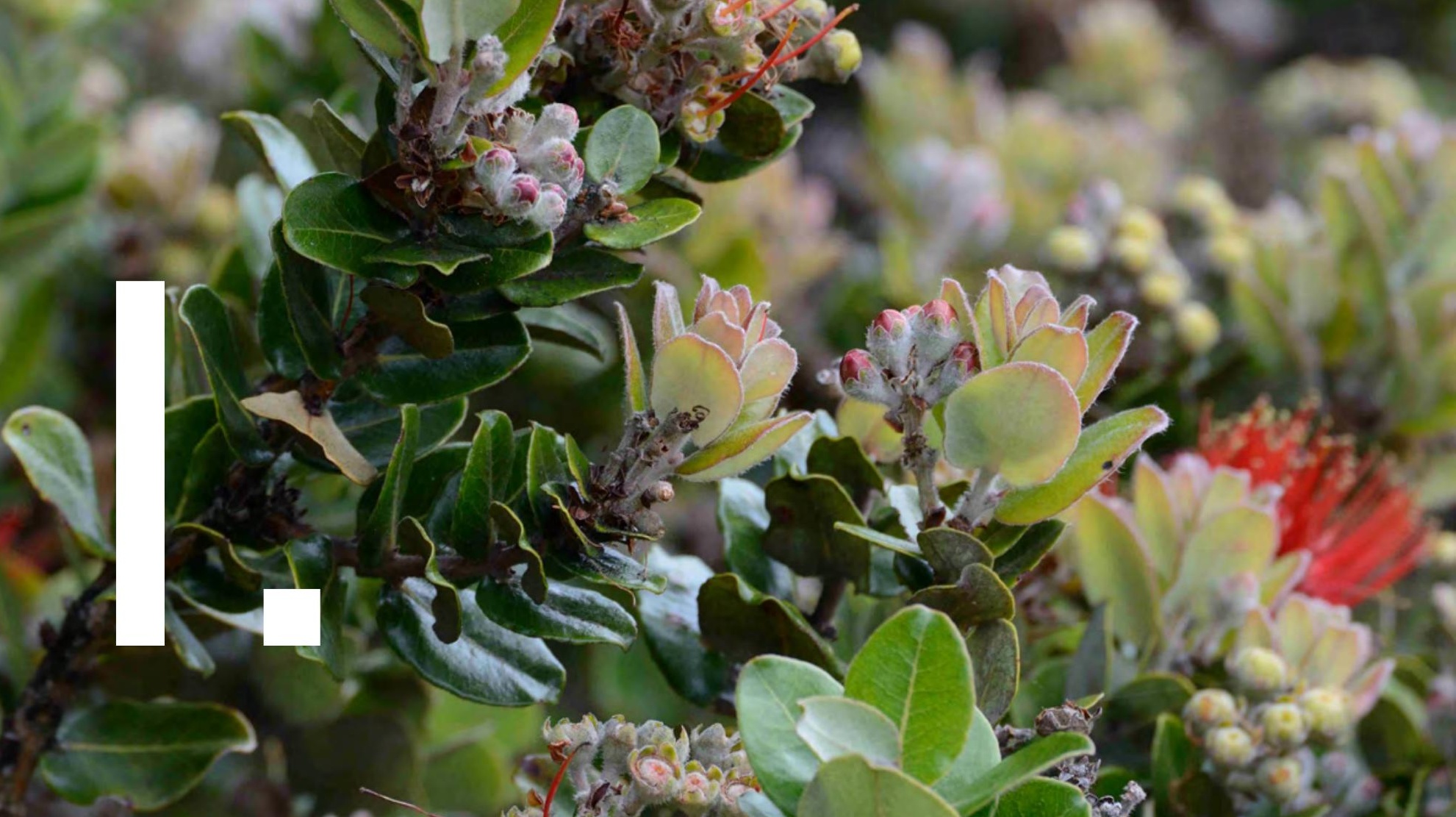




\section{Introducción}

El declive de muchas especies hacia la extinción ha motivado que los esfuerzos de conservación se centren en asegurar que las especies sigan existiendo. Sin embargo, los conservacionistas reconocen desde hace tiempo la necesidad de complementar esto con el objetivo de recuperar las poblaciones mermadas en toda el área de distribución de una especie y restaurar las especies en los ecosistemas de los que han sido extirpadas.

Reconociendo esta necesidad, la Unión Internacional para la Conservación de la Naturaleza (UICN) hizo un llamamiento a favor de la elaboración de criterios objetivos para las listas verdes de especies, ecosistemas y áreas protegidas (IUCN \& WCPA, 2017). En la Resolución WCC-2012-RES-41 del Congreso Mundial de la Naturaleza de 2012 se solicita que "la Comisión para la Supervivencia de las Especies (CSE)... lleve a cabo las consultas científicas internacionales necesarias para desarrollar unos criterios objetivos, transparentes y reproducibles para la elaboración de Listas Verdes que evalúen de forma sistemática el éxito en la conservación de las especies".

En respuesta, la Comisión para la Supervivencia de las Especies organizó, bajo los auspicios del Comité de la Lista Roja de la UICN, un Grupo de Trabajo sobre la Evaluación del Éxito de la Conservación con el fin de supervisar la elaboración de este Estándar. El Grupo de Trabajo creó un marco para medir la recuperación de especies y el impacto de la conservación (Akçakaya et al., 2018)르 , con el que se proponía la definición de especie totalmente recuperada basada en la viabilidad, la funcionalidad y la representación, y se definían cuatro indicadores para cuantificar la importancia de la conservación de una especie. Este marco se probó en una serie de taxones entre 2018 y 2021 con el fin de asegurar una amplia aplicabilidad, y los cambios resultantes se recogen en este Estándar.

Para asegurar la comprensión completa de las evaluaciones sobre el Estado Verde de las Especies de la UICN, es muy importante remitirse a las últimas versiones de todos los documentos siguientes:

1. Antecedentes y Directrices sobre el Estado Verde de las Especies de la UICN, que acompaña a este Estándar, y que se actualizará periódicamente (en adelante Antecedentes y Directrices);

2. Categorías y Criterios de la Lista Roja de la UICN (UICN, 2012a);

3. Directrices de Uso de las Categorías y Criterios de la Lista Roja de la UICN (UICN, 2019);

4. Directrices para el Uso de los Criterios de la Lista Roja de la UICN a Nivel Regional y Nacional (UICN 2012b).

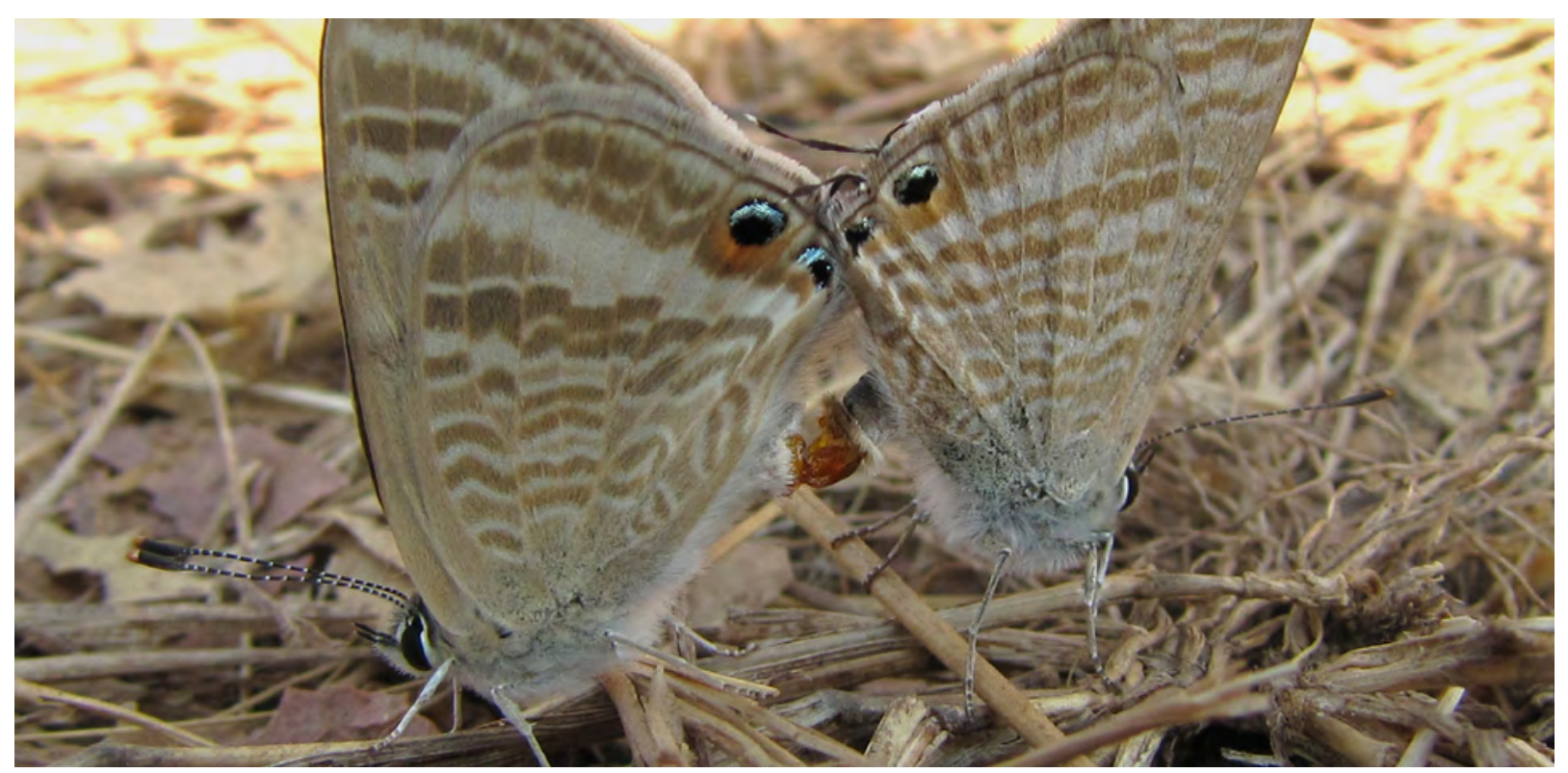

Apareamiento de la canela estriada (Lampides boeticus) @ Neha Mujumdar

1 Akçakaya et al. (2018) se considera la versión 1.0 del Estándar; el documento que está leyendo es la versión 2.0 y refleja los cambios realizados en respuesta a las pruebas y consultas que tuvieron lugar entre los años 2018 y 2020 


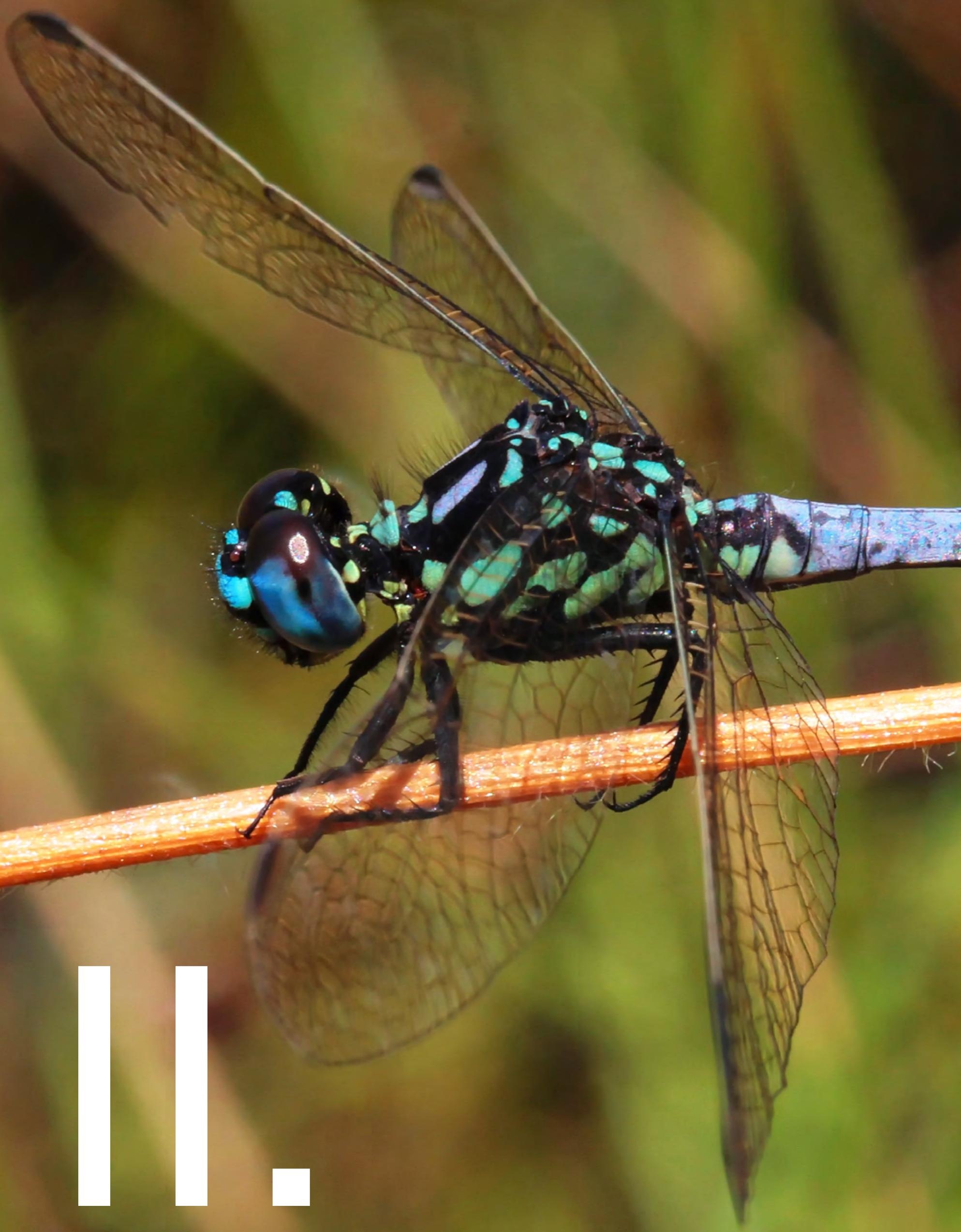




\section{Il. Preámbulo}

\section{Propósito}

El Estado Verde de las Especies de la UICN tiene cinco objetivos principales:

i. Proporcionar un marco normalizado para medir la recuperación de las especies;

ii. Reconocer los logros en materia de conservación;

iii. Poner de relieve las especies cuyo estado de conservación actual depende de la continuación de las acciones de conservación;

iv. Prever el impacto previsto en materia de conservación de las acciones de conservación planificadas; y

v. Elevar los niveles de ambición en términos de recuperación de especies a largo plazo.

Todos estos objetivos fomentan la conservación en aras de la recuperación de las especies, en toda su área de distribución. Se representan mediante una Puntuación de Recuperación de la Especie, y mediante cuatro indicadores de impacto de la conservación (Legado de conservación, Dependencia de la conservación, Ganancia de conservación, Potencial de recuperación), que se cuantifican como diferencias entre la Puntuación Verde de la especie en diferentes etapas a lo largo del tiempo o según diferentes escenarios.

\section{Alcance}

Las definiciones e indicadores del Estado Verde de las Especies pueden aplicarse a cualquier especie, excepto a los microorganismos. Las especies se pueden evaluar en el marco de este protocolo independientemente de su categoría en la Lista Roja y de si han sido objeto de medidas de conservación. Por razones prácticas (véase la sección V.3), el Estado Verde de una especie debe evaluarse después de la evaluación de la Lista Roja de esa especie o simultáneamente a ella.

\section{Recuperación de especies}

Según el presente estándar, el concepto de especie Totalmente recuperada se basa en la viabilidad, la funcionalidad y la representación (véase la definición en la sección III). La viabilidad es el primer requisito esencial, aunque no suficiente, para reconocer una especie como recuperada. Para que se considere Totalmente recuperada, una especie también debe mostrar sus interacciones ecológicas, funciones y otros roles en el ecosistema, y aparecer en un conjunto representativo de ecosistemas y comunidades en toda su área de distribución. Los aspectos de viabilidad y funcionalidad se examinan en la evaluación del estado de la población de la especie en cada unidad espacial (véanse las secciones IV.1, V.3.c y V.3.d), y el aspecto relativo a la representación se examina realizando la evaluación en todas las unidades espaciales del área de distribución de la especie (véanse las secciones IV.1 y V.2). La definición en base a estas características se utiliza para medir la recuperación de una especie, expresada como Puntuación Verde, que a su vez se utiliza para definir cuatro indicadores de impacto de la conservación concebidos para cuantificar la importancia de la conservación de la especie (véase la sección III, Definiciones).

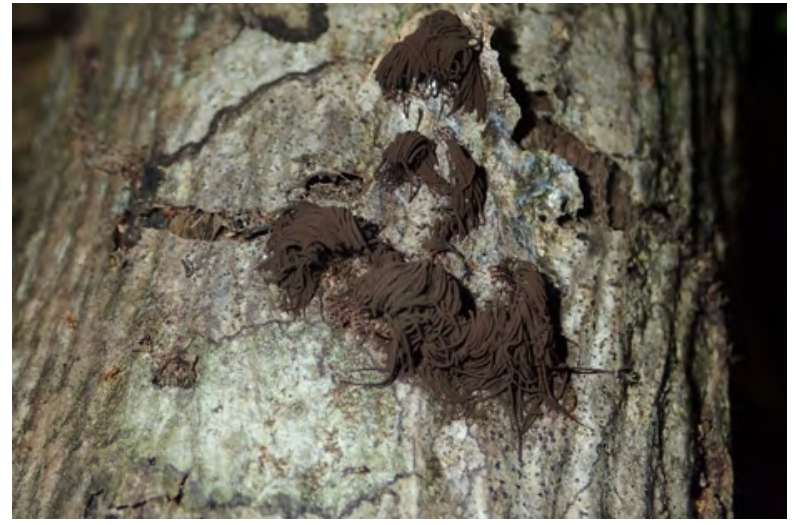

Stemonaria longa en Guadalupe (C) Alain Michaud

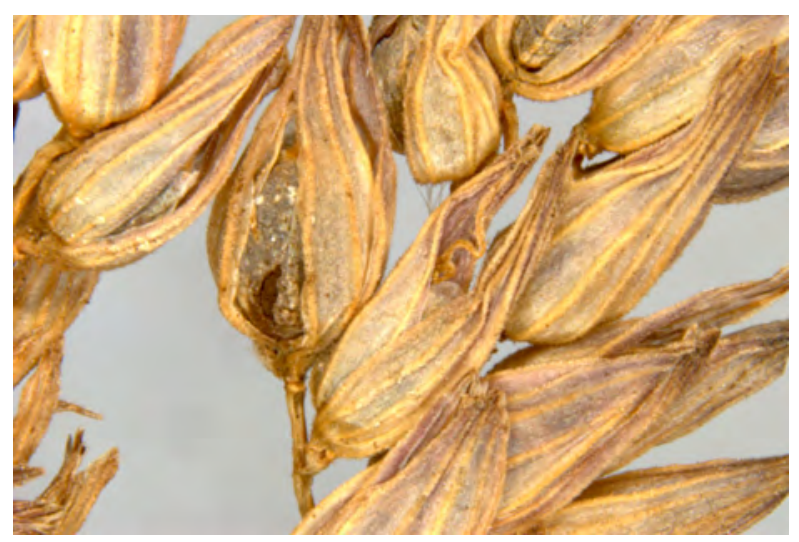

Ustilago suddiana (C) C. M. Denchev 


\section{Relación con la Lista Roja de la UICN}

Las evaluaciones del Estado Verde no son una alternativa a las evaluaciones del riesgo de extinción realizadas mediante la Lista Roja de la UICN, sino que proporcionan información complementaria. Los resultados de una evaluación del Estado Verde (puntuación y categoría relativas a la Recuperación de la Especie, y los indicadores y categorías de impacto de la conservación) deben considerarse junto con la categoría de las especies en la Lista Roja de la UICN. No existe una relación simple y general entre el estado de la Lista Roja y el Estado Verde de las Especies. Las especies que se han recuperado pueden seguir estando amenazadas; las especies que no se han recuperado pueden no estar amenazadas; y las especies con valores altos en los indicadores de impacto de la conservación pueden estar o no en riesgo de extinción. La Lista Roja y el Estado Verde proporcionan evaluaciones separadas, aunque relacionadas y complementarias del estado de conservación de una especie.

Algunas cuestiones que son relevantes para las evaluaciones del Estado Verde, por ejemplo, los términos utilizados para definir la viabilidad, se tratan en las Directrices de la Lista Roja; por lo tanto, los evaluadores deben consultar también la versión más reciente de las Directrices de uso de las Categorías y Criterios de la Lista Roja de la UICN (Subcomité de Estándares y Peticiones de la UICN, 2019), ya que se actualizan periódicamente.

\section{Relación con la planificación y las prioridades en materia de conservación}

Las evaluaciones del Estado Verde no tienen por objeto sustituir el proceso de establecimiento de objetivos, metas y propósitos en materia de recuperación, el cual forma parte del proceso de planificación de acciones de conservación que reúne a todos los interesados en el proceso de planificación, y que constituye el marco ideal y adecuado para establecer metas y prioridades de conservación. En lugar de ello, las evaluaciones del Estado Verde pretenden ser una parte útil e integral de este proceso de planificación, y reflejar los objetivos y aspiraciones que se establecen dentro de este proceso. Los planes de conservación o recuperación de especies suelen elaborarse con medidas y acciones de conservación que abarcan un horizonte temporal relativamente corto (por ejemplo, cinco o diez años); esto se refleja en el indicador Ganancia de conservación. Estos planes suelen estar enmarcados en una visión a más largo plazo, que a menudo puede abarcar entre 50 y 100 años. El indicador Potencial de recuperación está en consonancia con esta visión a largo plazo. El marco temporal del Potencial de recuperación se establece en 100 años, de modo que pueda vincularse de forma explícita con las declaraciones de principios de muchas estrategias y planes de acción de conservación. El Potencial de recuperación debería basarse, idealmente, en la declaración de principios a largo plazo de un proceso de planificación de acciones reconocido, que incluya una participación ponderada y adecuada de los interesados. Los procesos de planificación de la conservación también suelen basarse en acciones de conservación anteriores y en evaluaciones de los impactos de la conservación en diferentes partes del área de distribución de la especie, así como en las necesidades de conservación a corto plazo de esta última. Estos conceptos se recogen en los indicadores Legado de conservación y Dependencia de la conservación.

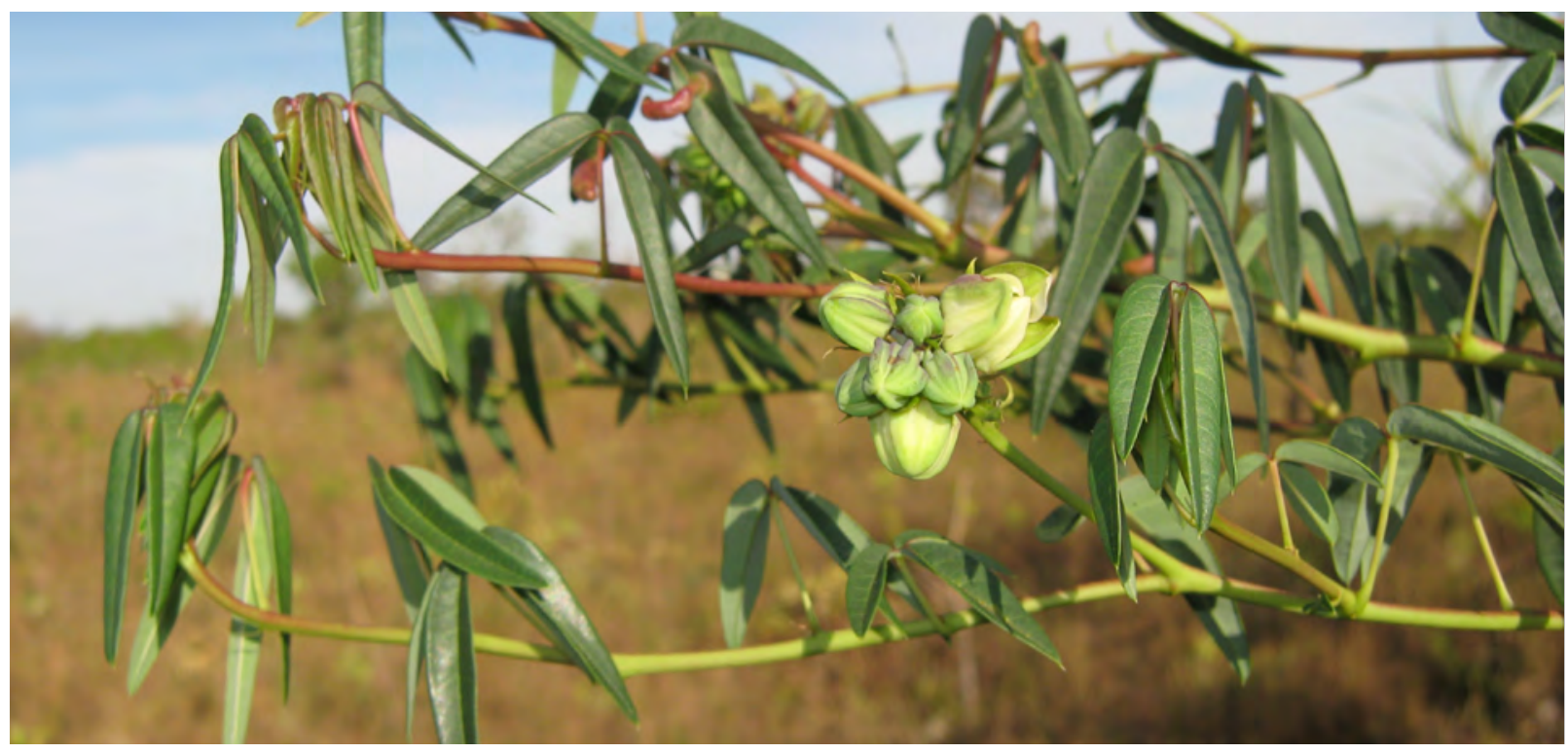

Manihot gracilis Pohl, un pariente silvestre secundario de la mandioca (M. esculenta subsp. esculenta), en el Brasil @ Marcelo Simon 


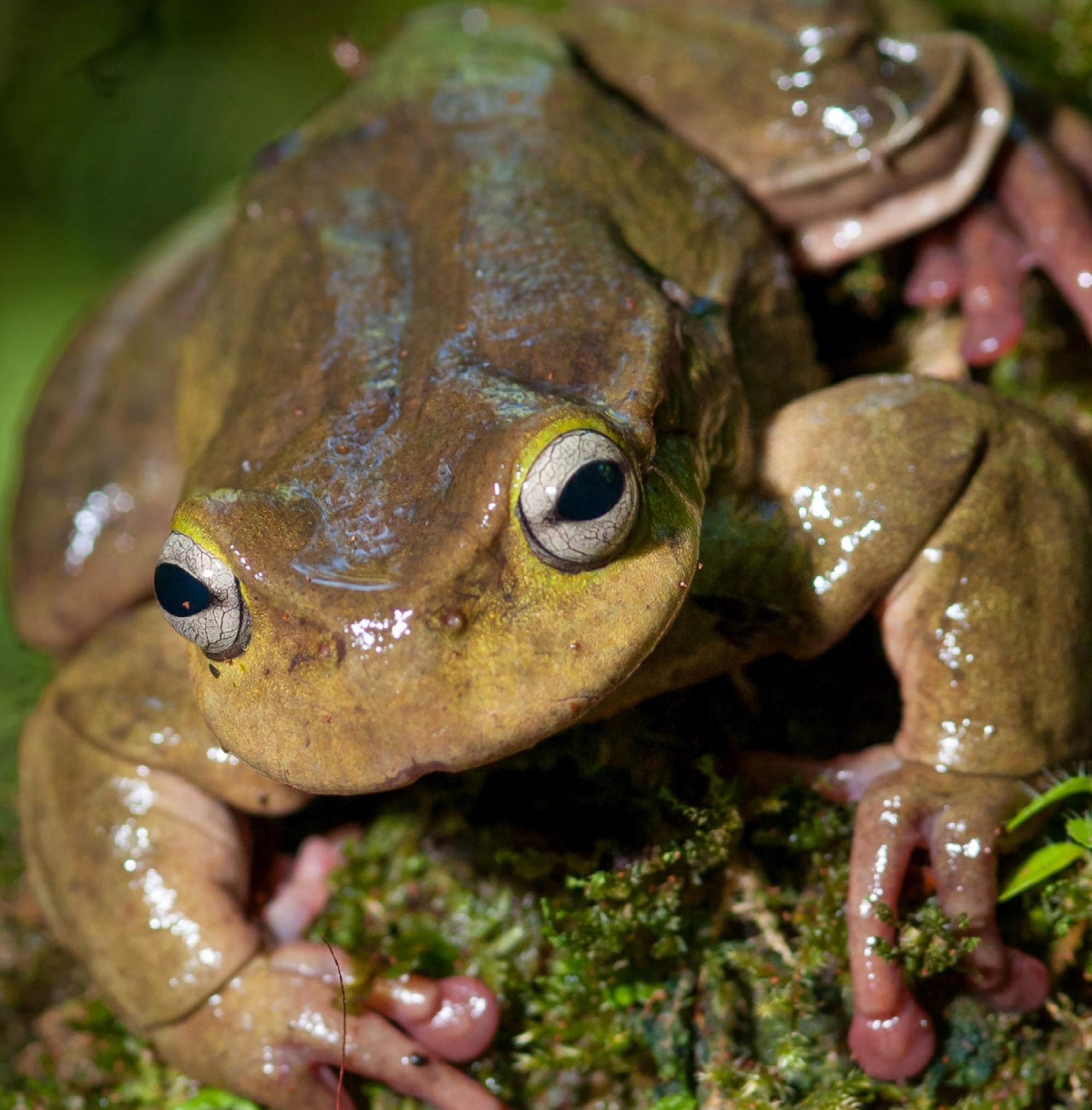

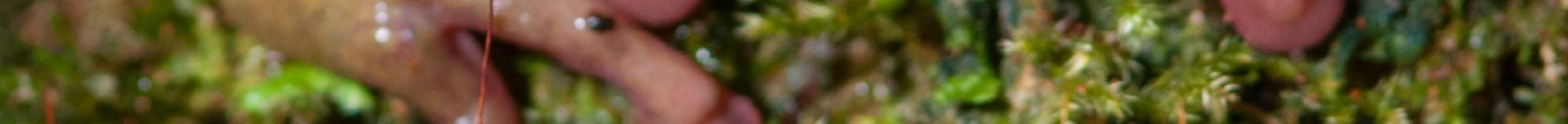

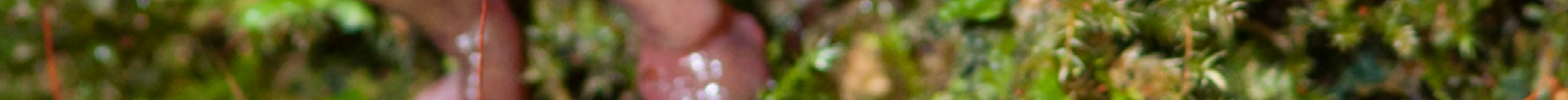
(1.

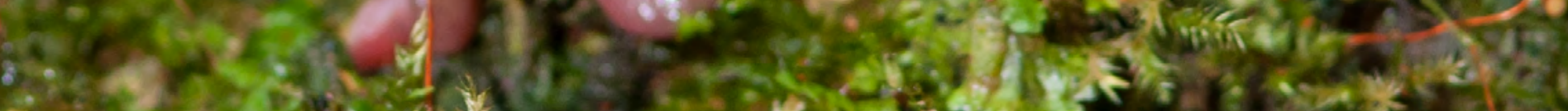

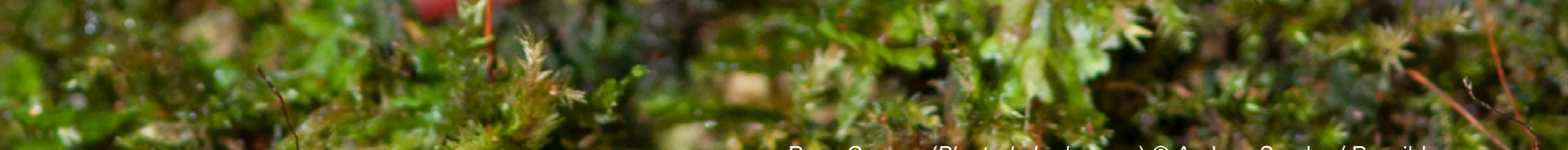
:

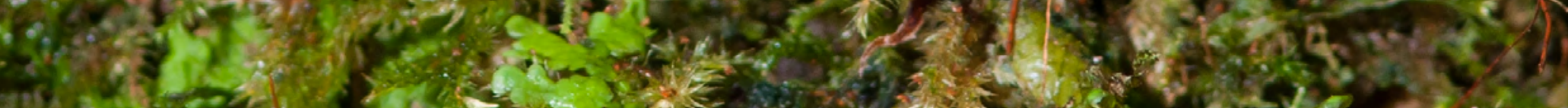



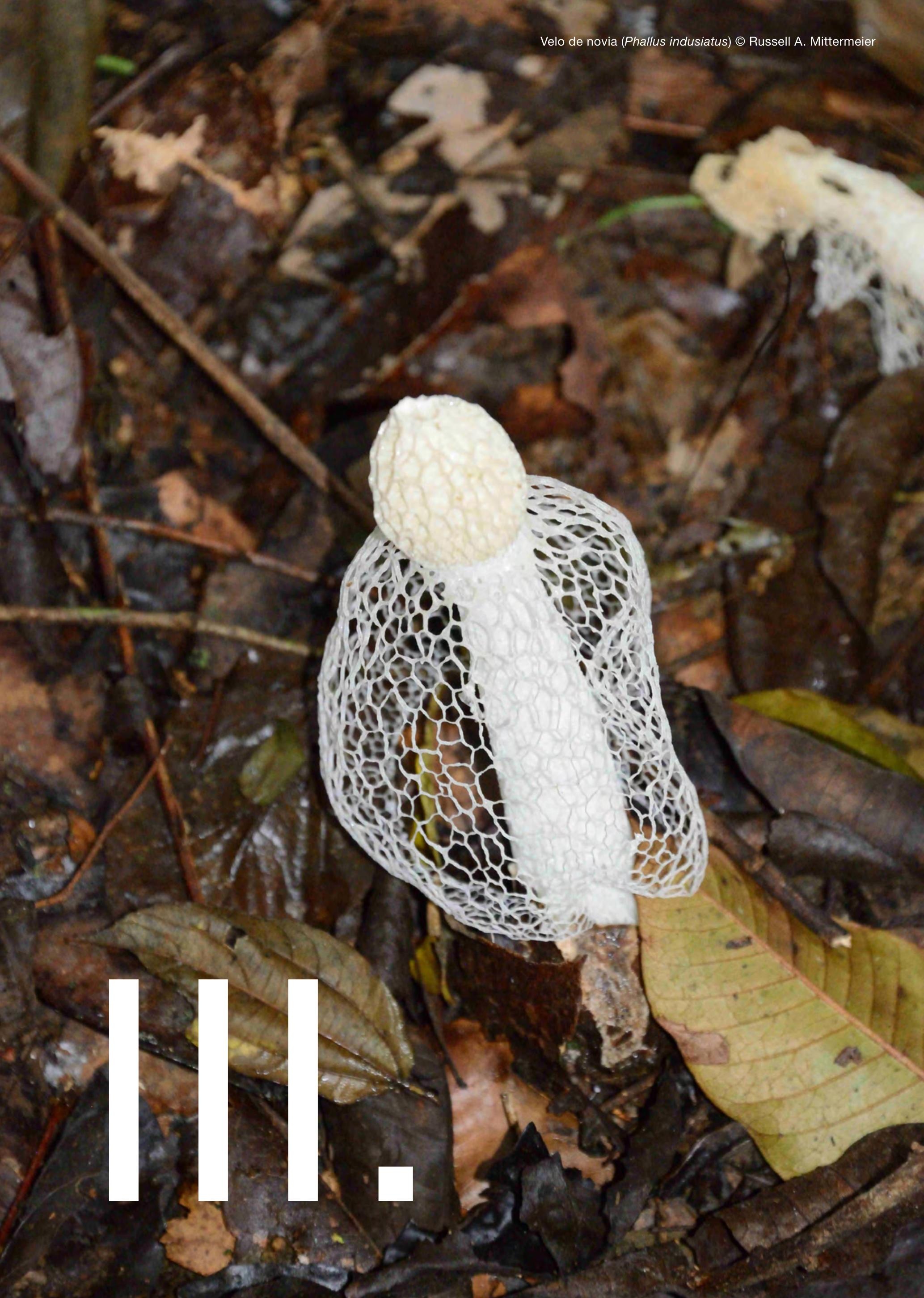


\begin{tabular}{ll}
\hline Ausente (estado de una unidad & Se dice que una especie está Ausente en una unidad espacial determinada si \\
espacial) & no se encuentra allí en estado silvestre, a pesar de que dicha unidad espacial \\
& forme parte del área de distribución de la especie. Se trata de uno de los \\
& cuatro estados posibles que puede asignarse a la población de una unidad \\
& espacial (además de Presente, Viable y Funcional).
\end{tabular}

Acción de conservación action

Cualquier actividad humana en la que la conservación de la biodiversidad sea una intención declarada, aunque la actividad no tenga como único o principal objetivo la conservación de la biodiversidad, y aunque su presupuesto provenga de fuentes distintas a los programas de conservación.

Dependencia de la conservación Indicador del impacto de la conservación que mide el impacto de las acciones de conservación en curso, que se define como el cambio previsto en la Puntuación Verde de la especie en el futuro a corto plazo (10 años) en caso de que, a partir de hoy, cesaran todas las acciones de conservación. El indicador se calcula como la diferencia entre la Puntuación Verde Actual (o la Referencia actual) y la Puntuación Verde Futuro sin conservación (véanse la sección IV.1, IV.2, V.5.c y la figura 1).

Ganancia de conservación

Indicador del impacto de la conservación que mide el impacto de las acciones de conservación en curso y planificadas, que se define como el cambio previsto en la Puntuación Verde de la especie en el futuro a corto plazo (10 años) en caso de que todas las acciones en curso y futuras planificadas se aplicaran de forma eficaz. El indicador se calcula como la diferencia entre la Puntuación Verde Actual (o la Referencia actual) y la Puntuación Verde Futuro con conservación (véanse la sección IV.1, IV.2, V.5.c y la figura 1).

Legado de conservación

Indicador del impacto de la conservación que mide el impacto de las acciones de conservación que se han llevado a cabo hasta la fecha, que se define como la diferencia entre la Puntuación Verde Actual de la especie y su Puntuación Verde Actual contrafactual (véanse la sección IV.1 y la figura 1).

Categoría de impacto de la conservación

Cada indicador de impacto de la conservación puede expresarse en forma de porcentaje o mediante categorías. Para cada uno de los cuatro indicadores de impacto de la conservación, existen las siguientes categorías: Negativa, Cero, Baja, Media, Elevada o Indeterminada (véase la sección IV.3).

Indicador de impacto de la conservación

Una de las cuatro formas de medir la importancia de las acciones de conservación para las especies: Legado de conservación; Dependencia de la conservación; Ganancia de conservación y Potencial de recuperación. Cada indicador se calcula como la diferencia entre dos puntuaciones verdes.

Contrafactual

Escenario hipotético de cuál habría sido el estado de la especie en la actualidad en caso de no haberse llevado a cabo acciones de conservación en el pasado; se utiliza para determinar el estado Actual contrafactual en cada unidad espacial, y la Puntuación Verde Actual contrafactual (véase la sección IV.1)

Función ecológica de una especie Totalidad de las interacciones de la especie, que determinan su influencia en los procesos de los ecosistemas o su contribución a ellos, y las pautas de las interacciones intraespecíficas, el comportamiento y la dinámica social que son característicos de esa especie (véase la sección V.3.d).

Funcionalidad ecológica de una población

Medida en que la población de una unidad espacial cumple la función o las funciones ecológicas de la especie en un momento y lugar concretos (por ejemplo, una unidad espacial), que se determina por su tamaño, densidad y estructura demográfica (véase la sección V.3.d).

Área de distribución

adicional prevista

Áreas que se espera firmemente que lleguen a ser adecuadas y habitadas por una especie en los próximos 100 años, teniendo en cuenta los cambios en el área de distribución resultantes del cambio climático y otros procesos globales y locales, así como las translocaciones para fines de conservación (Comité de Estándares y Peticiones de la UICN, 2019). 

Puntuación Verde se define en relación con esta condición. Esta definición y los indicadores de impacto de la conservación que se basan en ella se aplican no solo a las especies que han experimentado una disminución anteriormente, sino también a los taxones que no han disminuido (independientemente de si han sido o no objeto de conservación hasta el momento). Sin embargo, los taxones que se ajustan a esta definición pero que no se han beneficiado de la conservación se clasifican como No Mermados. Una especie Totalmente recuperada (o No mermada) tiene una Puntuación Verde del 100\%.

\section{Funcional (estado de una unidad espacial)}

Se dice que la población de una unidad espacial es Funcional si cumple las funciones ecológicas de la especie; es decir, tiene los atributos (incluidas, por ejemplo, la abundancia, la densidad y la estructura demográfica) que le permiten interactuar con otras especies, contribuir a los procesos de los ecosistemas y/o mostrar pautas de interacciones intraespecíficas, comportamientos y dinámicas sociales que son característicos de la especie. Se trata de uno de los cuatro estados posibles que se puede asignar a la población de una unidad espacial (además de Ausente, Presente y Viable). Véanse la sección V.3.d y la sección 4.5 Antecedentes y Directrices.

\section{Puntuación Verde}

Valor numérico entre el 0\% y el 100\%, que representa lo cerca que está la especie de estar Totalmente recuperada. Un valor del 0\% significa que la especie está Extinta o Extinta en Estado silvestre, y el 100\% significa que está Totalmente recuperada. La Puntuación Verde se calcula en función del estado (Ausente, Presente, Viable o Funcional) en cada unidad espacial, y puede calcularse respecto a períodos de tiempo pasados, actuales, futuros a corto plazo y futuros a largo plazo, y respecto a escenarios alternativos (es decir, con conservación a varios niveles (pasado, actual, planificado, aspirado), o sin conservación). Estas diferentes Puntuaciones Verdes se utilizan para calcular los indicadores de impacto de la conservación para cada especie, y las especies se ubican en categorías en función de estos cuatro indicadores.

\section{Área de distribución autóctona}

Partes del área de distribución

Presente (estado en una unidad espacial)

\section{Área de distribución}

Potencial de recuperación
Distribución de la especie, generada a partir de los registros actuales e históricos (escritos o verbales), o de las pruebas físicas de su presencia, dando cuenta de todos los sitios conocidos, inferidos o proyectados de presencia actual (UICN, 2012a), incluyendo las translocaciones pasadas para fines de conservación (Comité de Estándares y Peticiones de la UICN, 2019), pero sin incluir el vagabundeo. Cuando las pruebas directas son insuficientes para confirmar la ocupación anterior, la existencia de un hábitat adecuado dentro de una proximidad ecológicamente apropiada al área de distribución observada puede considerarse una prueba suficiente de dicha ocupación (CSE de la UICN, 2014).

Véase 'unidad espacial'.

Se dice que la población de una unidad espacial está Presente si la especie existe, pero no es Viable o Funcional en la unidad espacial. Se trata de uno de los cuatro estados posibles que se puede asignar a la población de una unidad espacial (además de Ausente, Viable y Funcional).

Distribución espacial de las especies, que comprende su área de distribución autóctona y su área de distribución adicional prevista. En cuanto al Legado de conservación, el área de distribución se define únicamente como el área de distribución autóctona. En cuanto a los otros tres indicadores de impacto de la conservación, que tienen que ver con el futuro de las especies, el área de distribución puede definirse como la combinación de su área de distribución autóctona y el área de distribución adicional prevista, dependiendo de cuándo se espera que se ocupe esta última. Véase la sección V.1.b.

Indicador de impacto de la conservación que cuantifica la aspiración o la ambición en términos de conservación, que se define como la máxima mejora plausible del estado de la especie con esfuerzos sostenidos de conservación e innovaciones en materia de conservación a largo plazo (100 años). Véanse la sección IV.1 y la figura 1. 

debido a los propágulos que inmigran desde otras unidades espaciales. Un propágulo es cualquier entidad viviente capaz de dispersarse y de producir un nuevo individuo maduro (p. ej., una espora, semilla, fruto, huevo, larva, parte de un individuo o la totalidad de este). Los gametos y el polen no están considerados como propágulos dentro de este contexto (UICN, 2012b).

Unidad espacial

Subdivisión del área de distribución de la especie en la que el estado de la especie se evalúa como Ausente, Presente, Viable o Funcional. Véase 'Delimitación de unidades espaciales' en la sección $V$, a continuación.

Población de una unidad espacial

Conjunto de individuos de una especie en una unidad espacial (véase "población" en UICN (2012a) y UICN (2019)). También se denomina "población en una unidad espacial".

\section{Categoría de Recuperación de la Especie}

\section{Puntuación de Recuperación de la Especie}

Medida que indica lo cerca que está la especie de estar Totalmente recuperada, según la Puntuación de Recuperación de la Especie y los umbrales indicados en la sección IV.3.

Medida que indica lo cerca que está la especie de estar Totalmente recuperada, según el estado observado, estimado, inferido o sospechado de la especie en el momento de la evaluación (en la figura 1 se muestra como la Puntuación Verde Actual). Puede trazarse en función del tiempo, basándose en el estado en cada etapa, representando el progreso (o no) de la especie hacia la recuperación total.

Estado

Condición de la especie en una unidad espacial, evaluada como una de las cuatro categorías ordinales: Ausente, Presente, Viable y Funcional. A estas categorías se les asignan ponderaciones (véase la sección V.3), que se combinan, utilizando la ecuación 1 que figura a continuación, para obtener la Puntuación Verde. El estado puede determinarse con respecto a períodos de tiempo pasados, actuales, futuros a corto plazo y futuros a largo plazo, así como con respecto a escenarios alternativos (con y sin conservación). Los estados se encuentran en una jerarquía anidada, de modo que una población de unidad espacial que se evalúa como Viable también está Presente por definición, y una población de unidad espacial que se evalúa como Funcional también es por definición Viable y Presente. Por lo tanto, solo se puede asignar la puntuación Funcional a las unidades espaciales que sean a la vez Viables y Funcionales (de todas formas, véase la sección V.3.d).

\section{Subpoblación}

Las subpoblaciones se definen como grupos de la población diferenciados entre sí por razones geográficas o por otros factores y entre los que existe escaso intercambio genético o demográfico (por regla general, como máximo una migración con éxito de un individuo o gameto al año)" (UICN, 2012a; véase Comité de Estándares y Peticiones de la UICN (2019) a modo de orientación).

Viable (estado en una unidad espacial)
Se dice que la población de una unidad espacial es Viable si el riesgo de extirpación de la especie en la unidad espacial es bajo, según la categoría de la Lista Roja regional de la UICN. Véase la sección V.3 para más detalles. 


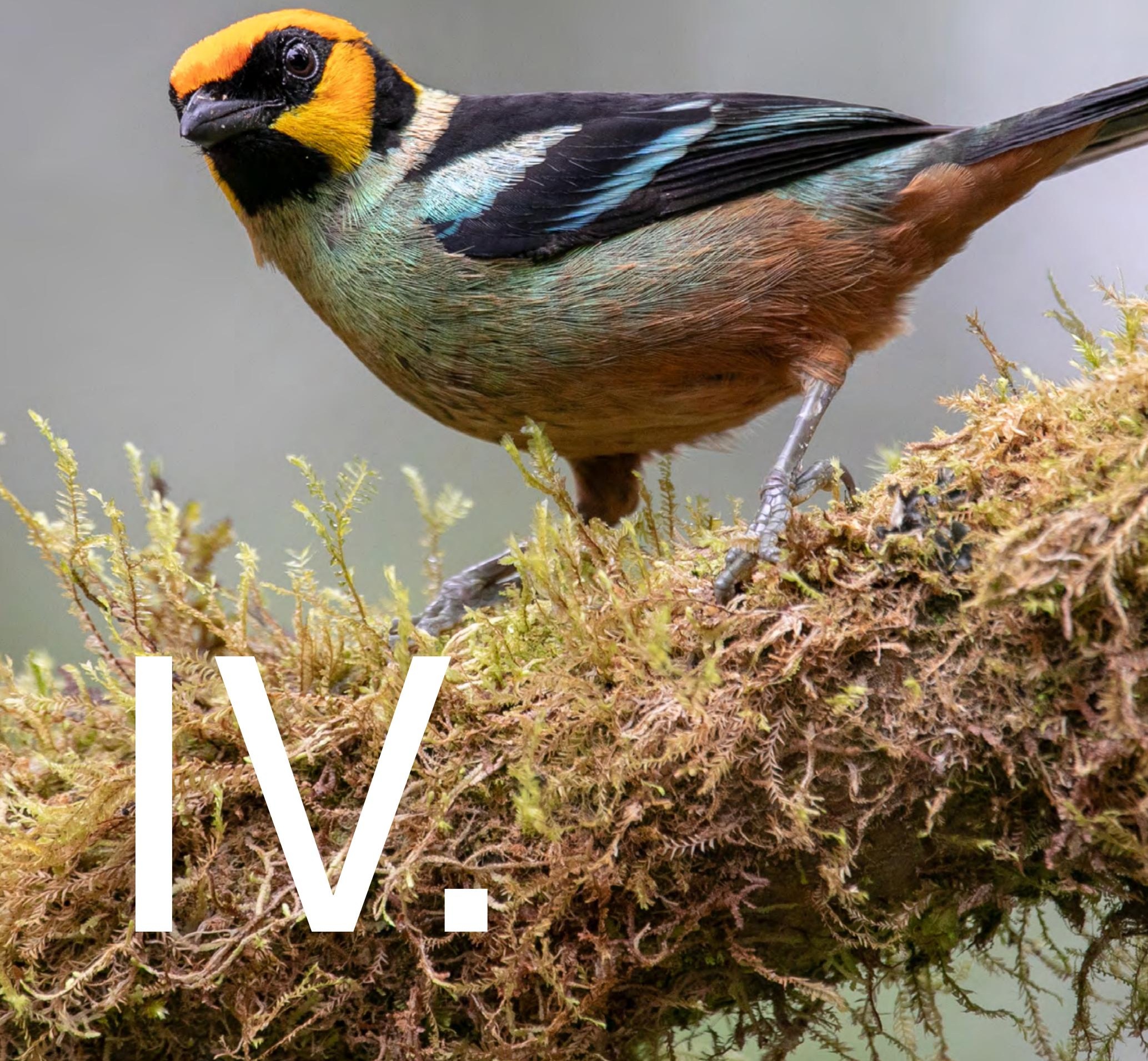




\section{Criterios}

\section{Puntuación Verde}

El estado de una especie en una unidad espacial se evalúa como una de las cuatro categorías ordinales: Ausente, Presente, Viable y Funcional (véase la sección V.3. para más detalles). Para obtener una Puntuación Verde (G) para la especie, basada en los estados en todas las unidades espaciales, se utiliza la ecuación (ecuación 1):

$$
G=\frac{\sum_{s} W_{s}}{W_{F} \times N} \times 100
$$

donde s es cada unidad espacial, $W_{S}$ la ponderación del estado (Ausente, Presente, Viable o Funcional) en la unidad espacial, $W_{F}$ es la ponderación del estado Funcional y $\mathrm{N}$ es el número de unidades espaciales. El denominador es la máxima puntuación posible que se alcanza cuando todas las unidades espaciales se evalúan como Funcionales. Por lo tanto, la Puntuación Verde se calcula como un porcentaje de la categoría Totalmente recuperada. Por lo que respecta a las puntuaciones Actual y Actual contrafactual, el denominador se basa en el número de unidades espaciales en el área de distribución autóctona únicamente (sin incluir el área de distribución adicional prevista).

Las diferentes Puntuaciones Verdes se calculan en función de los períodos de tiempo actuales, futuros a corto plazo y futuros a largo plazo, así como de los escenarios alternativos, tal como se indica en el cuadro 1. Los indicadores del impacto de la conservación (por ejemplo, el Legado de conservación, etc.) se calculan como diferencias entre dos Puntuaciones Verdes, como se detalla en la sección $\mathrm{V}$.

Cuadro 1. Nombres y descripciones de los escenarios conforme a los cuales se puede calcular la Puntuación Verde. Obsérvese que todas las evaluaciones del Estado Verde requieren al menos el escenario "Actual"; la evaluación del impacto de la conservación requiere al menos otro escenario.

\section{Puntuación Verde Escenario y período de tiempo}

Actual Puntuación Verde en el momento de la evaluación (la misma que la Puntuación de Recuperación de la Especie en ese momento).

Actual contrafactual

Valor que tendría la Puntuación Verde actualmente si no se hubieran llevado a cabo acciones de conservación en el pasado. Véase la sección V.4.

Referencia actual

Valor previsto de la Puntuación Verde en el futuro a corto plazo (10 años), teniendo en cuenta los beneficios probables de las acciones de conservación que se están llevando a cabo actualmente o que es muy probable que se lleven a cabo dentro de 1 año. Si no se especifica el escenario de Referencia actual, se supondrá que es el mismo que en el caso de Actual. Véase la sección V.5.c.

Futuro con conservación $\quad$ Valor previsto de la Puntuación Verde en el futuro a corto plazo (10 años), teniendo en cuenta los beneficios probables de las acciones de conservación que se están llevando a cabo actualmente o cuya aplicación está prevista durante este período de tiempo. Véase la sección V.5.a.

Futuro sin conservación

Valor previsto de la Puntuación Verde en el futuro a corto plazo (10 años), suponiendo que las acciones de conservación en curso se detuvieran hoy y no se aplicaran nuevas acciones. Véase la sección V.5.b.

Potencial a largo plazo 


\section{Indicadores de impacto de la conservación}

Para cualquier especie, se calculan cuatro indicadores de impacto de la conservación como resultado de la diferencia entre dos Puntuaciones Verdes (véase la figura 1):

\section{Legado de conservación = Actual - Actual contrafactual}

(se mide el impacto de las acciones de conservación realizadas hasta la fecha)

\section{Dependencia de la conservación = Referencia actual - Futuro sin conservación Ganancia de conservación = Futuro con conservación - Referencia actual}

(se mide tanto el efecto futuro a corto plazo de las acciones de conservación en curso como las planificadas)

Potencial de recuperación $=$ Potencial a largo plazo - Actual

(se mide el efecto futuro a largo plazo del esfuerzo de conservación plausible y de la innovación)

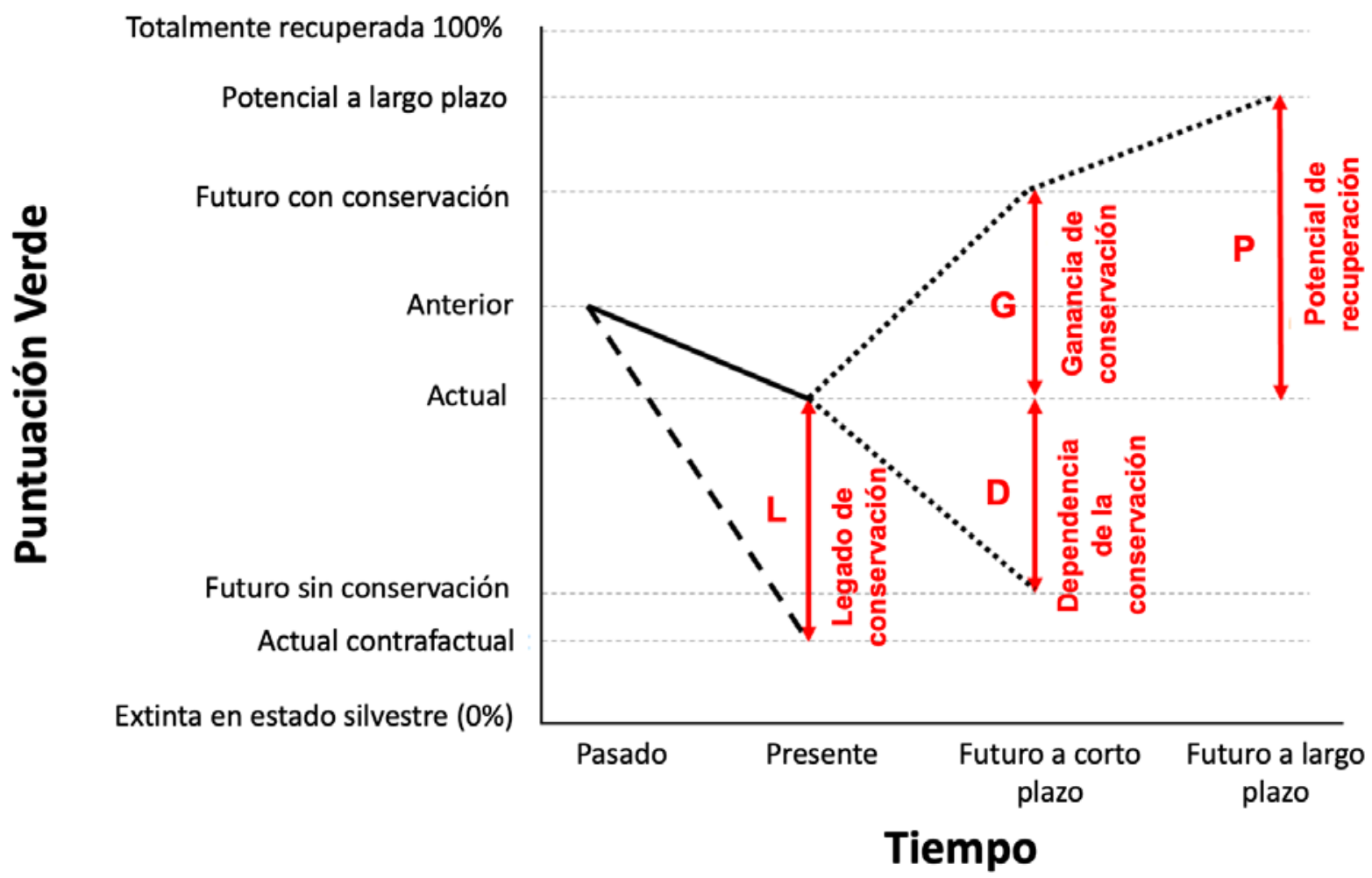

Figura 1. Representación gráfica de los indicadores de impacto de la conservación a modo de diferencias en el grado de recuperación de la especie (porcentaje de Totalmente recuperada, medido como la Puntuación Verde; ecuación 1). Línea negra continua: cambio observado en la Puntuación Verde de la especie. Línea negra de guiones: (contrafactual) cambio pasado previsto en ausencia de esfuerzos de conservación pasados. Líneas negras punteadas: escenarios futuros de cambio previstos con y sin los esfuerzos de conservación actuales y futuros. Las flechas verticales representan los indicadores de impacto de la conservación: Legado de conservación: beneficios de la conservación actual y pasada (actual - actual contrafactual); Dependencia de la conservación: cambio previsto en el futuro a corto plazo en ausencia de conservación en curso (referencia actual - futuro sin conservación); Ganancia de conservación: mejora prevista en el futuro a corto plazo con la conservación en curso y planificada (futuro con conservación - referencia actual); Potencial de recuperación: posible mejora con la conservación a largo plazo (potencial a largo plazo - actual). En este ejemplo, se supone que la Referencia actual (véase la sección V.5.c) tiene el mismo valor que la puntuación Actual; véase Antecedentes y Directrices para ver el mismo gráfico con una hipótesis alternativa. 


\section{Categorías y umbrales}

A efectos de la mayoría de los casos, se deben comunicar los valores numéricos (porcentaje) de la Puntuación de Recuperación de la Especie y los cuatro indicadores de impacto de la conservación, incluidas sus incertidumbres (véase la sección V.8, más adelante). Sin embargo, los resultados por categorías pueden ser más apropiados para algunos usos y grupos de destinatarios (véase la sección V.8). Por lo tanto, cada indicador se divide en categorías, con el fin de i) proporcionar un contexto y permitir la interpretación del valor porcentual del indicador, y ii) destacar los casos de impacto, dependencia o potencial de conservación sobresalientes.

La Puntuación de Recuperación de la Especie (PRE) puede presentarse de forma numérica (desde el 0\% para Extinta hasta el 100\% para Totalmente recuperada), o por categorías. Las categorías de Recuperación de la Especie se basan en los valores de mejor estimación, mínimo y máximo de $\mathrm{PRE}\left(\mathrm{PRE}_{\text {mejor’ }}, \mathrm{PRE}_{\text {mím }}, \mathrm{PRE}_{\text {máx }}\right.$, respectivamente) y el valor de mejor estimación de Legado de conservación ( $L_{\text {mejor }}$ ), y se asignan de acuerdo con las siguientes reglas, que se aplican en el orden indicado hasta que se cumple la condición dada para una categoría (cuadro 2).

Cuadro 2. Categorías de Recuperación de la Especie, basadas en los valores de mejor estimación, mínimo y máximo de la Puntuación de Recuperación de la Especie $\left(P R E_{\text {mejor }}, \mathrm{PRE}_{\text {mín }}\right.$, $\mathrm{PRE}_{\text {máx }}$, respectivamente):

\begin{tabular}{|ll|}
\hline Indeterminada & Si $\left(P R E_{\text {máx }}-P R E_{\text {min }}\right)>40 \%$ \\
\hline No mermada & Si $\left(P R E_{\text {mejor }}=100 \%\right)$ y $\left(L_{\text {mejor }}=0 \%\right)$ \\
\hline Totalmente recuperada & Si PRE $E_{\text {mejor }}=100 \%$ \\
\hline Ligeramente mermada & Si PRE $E_{\text {mejor }}>80 \%$ \\
\hline Moderadamente mermada & Si PRE $E_{\text {mejor }}>50 \%$ \\
\hline Mayormente mermada & Si PRE $E_{\text {mejor }}>20 \%$ \\
\hline Gravemente mermada & If $\mathrm{SRS}_{\text {best }}>0 \%$ \\
\hline Extinta en estado silvestre & If $\mathrm{SRS}_{\text {best }}=0 \%$ \\
\hline
\end{tabular}


La categoría correspondiente a cada uno de los cuatro indicadores de impacto de la conservación se determina en función de las condiciones indicadas a continuación (cuadros 3-6). Para cada indicador, las reglas se aplican en el orden indicado hasta que se cumple la condición dada para una categoría. En estas condiciones, se utilizan los valores de mejor estimación, mínimo y máximo de los indicadores de impacto de la conservación y los valores de mejor estimación de las Puntuaciones Verdes (como Actual y Actual contrafactual). Para cada indicador, la categoría Elevada puede alcanzarse de una de estas tres maneras: el valor numérico es superior al 40\%; el valor numérico es bajo, pero representa la evitación de la extinción; o el valor numérico es bajo, pero es sustancial en comparación con la mejor o mediana estimación de la puntuación Actual. Para obtener información sobre los umbrales, consulte Antecedentes y Directrices.

Cuadro 3. Categorías de Legado de conservación, basadas en los valores de mejor estimación, mínimo y máximo del indicador de Legado de conservación $\left(L_{\text {mejor }}, L_{\text {min }}\right.$, $L_{\text {máx }}$, respectivamente):

\begin{tabular}{|ll|} 
Indeterminada & Si $\mathrm{L}_{\text {min }}<0 \%$ y $\mathrm{L}_{\text {máx }}>40 \%$ \\
\hline Elevada & Si $\mathrm{L}_{\text {mejor }}>40 \%$, o \\
& $\begin{array}{l}\mathrm{L}_{\text {mejor }}>0 \% \text { y Actual contrafactual }=0 \text { (es decir, se evita la extinción), o } \\
L_{\text {mejor }}>1 / 2 \cdot \text { Actual (es decir, un legado sustancial relativo a la puntuación actual) }\end{array}$ \\
\hline Media & Si $L_{\text {mejor }}>10 \%$ \\
\hline Baja & Si $L_{\text {mejor }}>0 \%$ \\
\hline Cero & Si $L_{\text {mejor }}=0 \%$ \\
\hline Negativa & Si $L_{\text {mejor }}<0 \%$ \\
\hline
\end{tabular}

Nota: el carácter · corresponde al operador matemático de la multiplicación (igual que $\times$ )

Cuadro 4. Categorías de Dependencia de la conservación, basadas en los valores de mejor estimación, mínimo y máximo del indicador de Dependencia de la conservación $\left(D_{\text {mejor }}, D_{\text {minn }}, D_{\text {máx }}\right.$, respectivamente):

Indeterminada $\quad$ Si $D_{\text {min }}<0 \%$ y $D_{\text {máx }}>40 \%$

\begin{tabular}{|c|c|}
\hline Elevada & $\begin{array}{l}\text { Si } D_{\text {mejor }}>40 \% \text {, o } \\
D_{\text {mejor }}>0 \% \text { y Futuro sin conservación=0 (es decir, se extinguiría sin conservación), o } \\
D_{\text {mejor }}>1 / 2 \cdot \text { Actual (o Referencia actual) (es decir, dependencia sustancial relativa a la } \\
\text { puntuación actual) }\end{array}$ \\
\hline Media & Si $D_{\text {mejor }}>10 \%$ \\
\hline Baja & Si $D_{\text {mejor }}>0 \%$ \\
\hline Cero & Si $D_{\text {mejor }}=0 \%$ \\
\hline Negativa & Si $D_{\text {mejor }}<0 \%$ \\
\hline
\end{tabular}


Cuadro 5. Categorías de la Ganancia de conservación, basadas en los valores de mejor estimación, mínimo y máximo del indicador de Ganancia de conservación $\left(G_{m e j o r}, G_{\text {min }}, G_{\text {máx }}\right.$, respectivamente):

Indeterminada $\quad \mathrm{Si} \mathrm{G}_{\text {min }}<0 \%$ y $\mathrm{G}_{\text {máx }}>40 \%$

Elevada $\quad \mathrm{Si} \mathrm{G}_{\text {mejor }}>40 \%$, o

$\mathrm{G}_{\text {mejor }}>0 \%$ y Actual=0 (es decir, seguiría teniendo la categoría EW sin conservación), o

$\mathrm{G}_{\text {mejor }}>1 \cdot$ Actual (o Referencia actual) (es decir, recuperación sustancial relativa a la

puntuación actual)

Media $\quad$ Si $G_{\text {mejor }}>10 \%$

Baja $\quad$ Si $G_{\text {mejor }}>0 \%$

Cero $\quad \mathrm{Si} \mathrm{G}_{\text {mejor }}=0 \%$

Negativa $\quad \mathrm{Si} \mathrm{G}_{\text {mejor }}<0 \%$

Cuadro 6. Categorías del Potencial de recuperación, basadas en los valores de mejor estimación, mínimo y máximo del indicador Potencial de recuperación $\left(P_{\text {mejor }}, P_{\text {min }}, P_{\text {máx }}\right.$, respectivamente):

Indeterminada $\quad$ Si $P_{\text {min }}<0 \%$ y $P_{\text {máx }}>40 \%$

Elevada $\quad$ Si $P_{\text {mejor }}>40 \%, 0$

$P_{\text {mejor }}>0 \%$ y Actual=0 (es decir, seguiría teniendo la categoría EW sin conservación), o

$P_{\text {mejor }}>2 \cdot$ Actual (es decir, recuperación sustancial relativa a la puntuación actual)

Media $\quad$ Si $P_{\text {mejor }}>10 \%$

Baja $\quad$ Si $P_{\text {mejor }}>0 \%$

Cero $\quad$ Si $P_{\text {mejor }}=0 \%$

Negativa $\quad$ Si $P_{\text {mejor }}<0 \%$ 
Campánulas (Campanula mugeana) of Hasan Yildın

$7 \times 2 \times 3 \times 5$

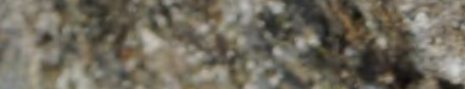

Whes of

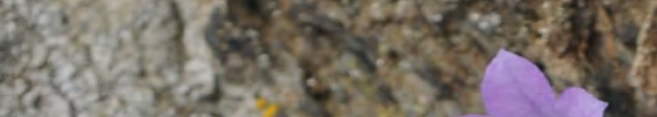

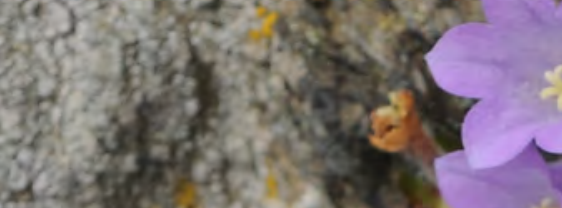

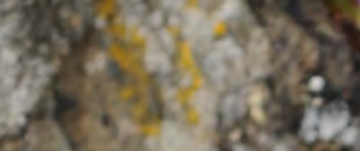

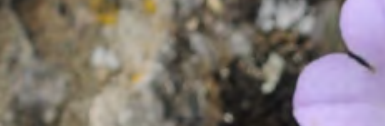

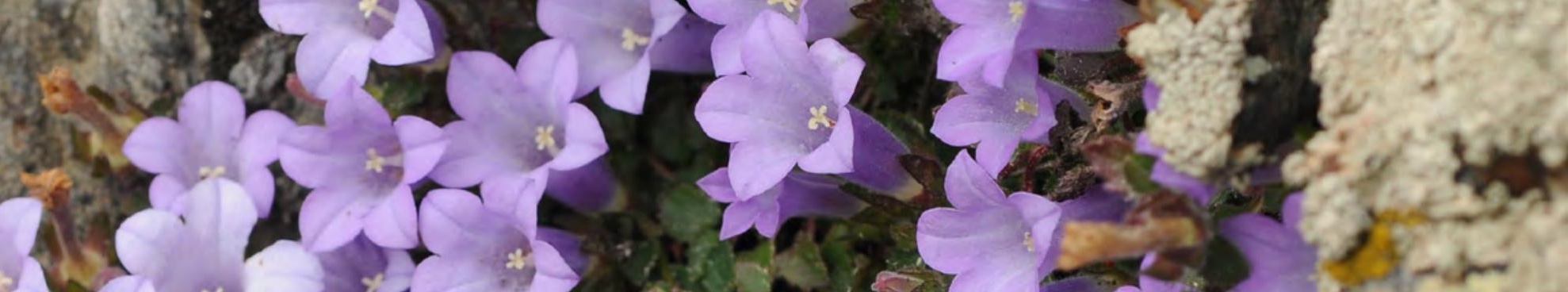
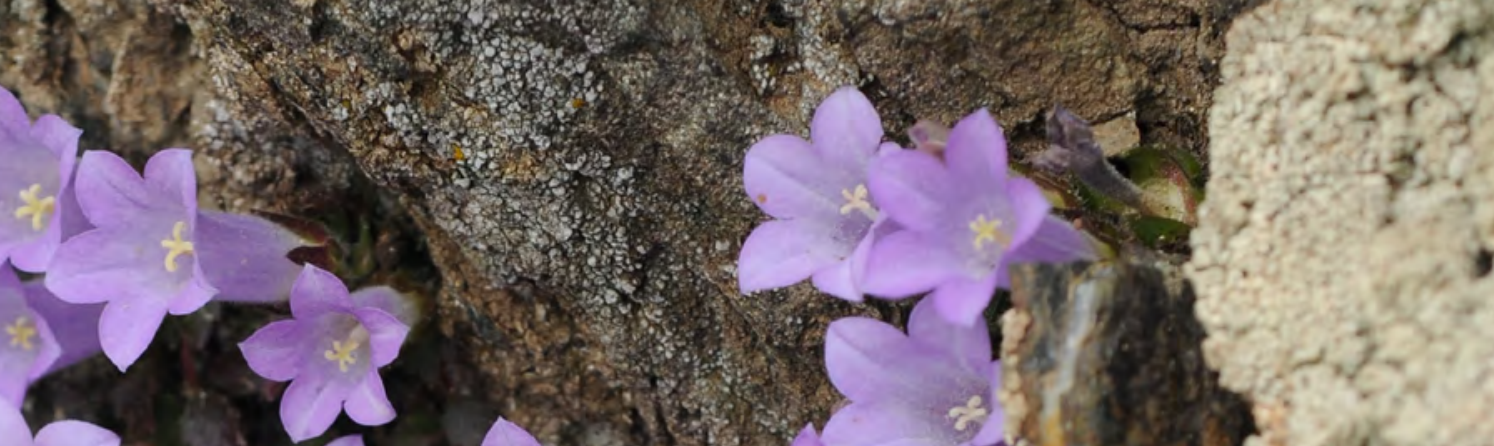

$y$

w

(1)

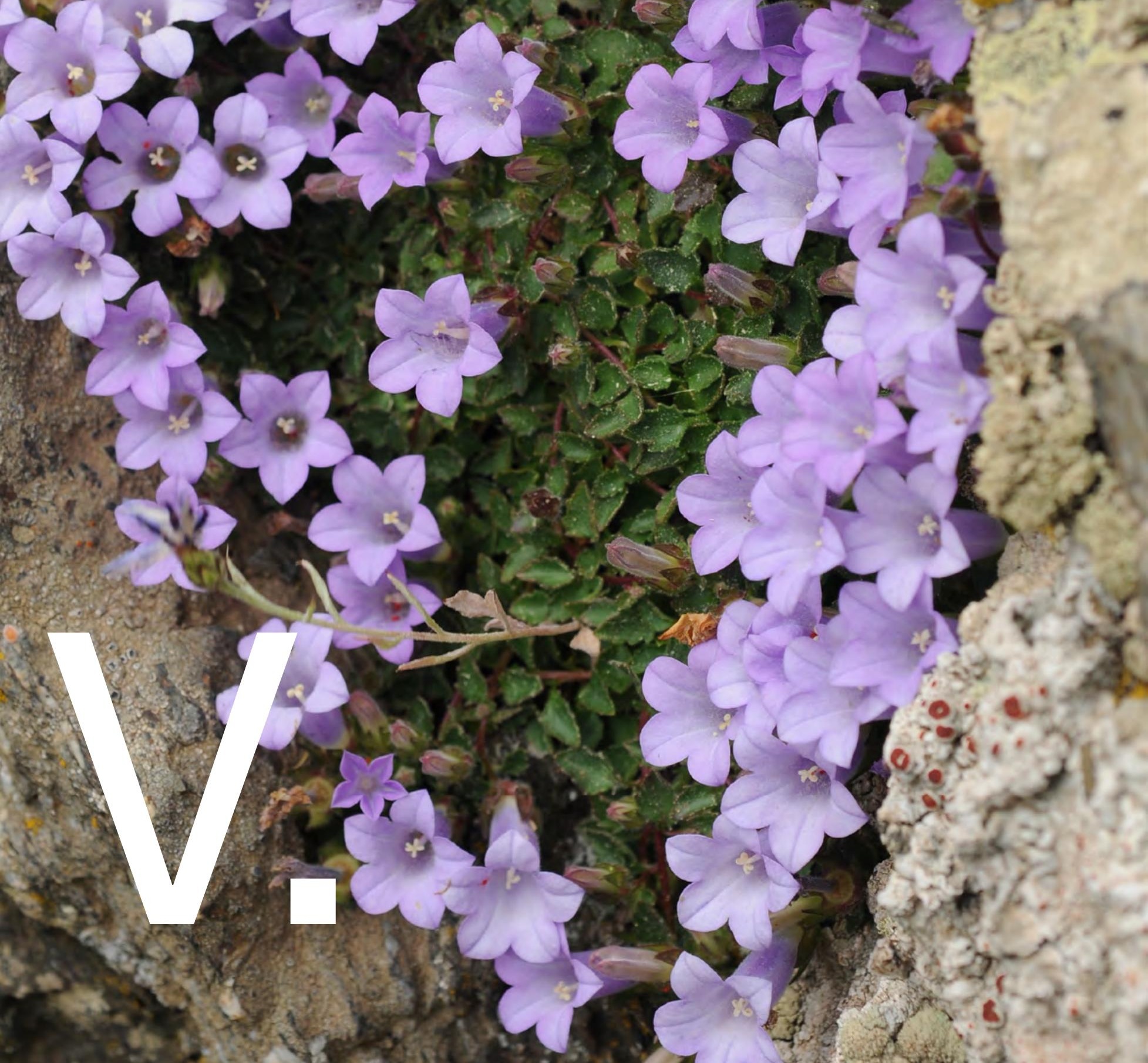




\section{Procedimientos}

Los siguientes procedimientos son los principales componentes de una evaluación del Estado Verde. Estos procedimientos se discuten en detalle en los Antecedentes y Directrices relativos al Estado Verde de las Especies de la UICN, que se irán modificando con el tiempo para incorporar orientaciones sobre el uso de los nuevos tipos de datos que puedan estar disponibles, y los nuevos avances en los métodos de análisis.

\section{Determinación del área de distribución}

\section{a. Determinación del área de distribución autóctona}

El área de distribución autóctona (véase la definición) se basa en todos los lugares de presencia conocidos, inferidos y (espacialmente) proyectados, tanto actuales como históricos. Incluye las translocaciones para fines de conservación que se ajustan al Subcomité de Estándares y Peticiones de la UICN (2019), pero no incluye el vagabundeo. Para que los objetivos de recuperación sean ambiciosos, y para evitar que se modifiquen las pautas de referencia, el área de distribución autóctona debe basarse en la distribución en la fecha más temprana que sea posible, reconociendo al mismo tiempo que si se retrocede demasiado aumentarán las incertidumbres debido a la escasez de datos. Lo ideal sería que esta fecha se fijara en "una época anterior a que el ser humano fuera el elemento más importante de limitación de las distribuciones de las especies" (Sanderson, 2019). Sin embargo, las diferencias entre las especies en cuanto a la disponibilidad y la calidad de los datos hacen que ese criterio dé lugar a evaluaciones difícilmente comparables. Por lo tanto, las especies que se encuentran en la misma región geográfica y bajo amenazas similares deberían tener fechas de referencia similares para determinar el área de distribución autóctona (Stephenson et al. 2019). Partiendo del análisis de las tendencias de los datos históricos sobre la cobertura de los hábitats y las poblaciones humanas (de Klein Goldewijk et al., 2011), en la mayoría de las regiones resulta adecuado utilizar como fecha de referencia por defecto el año 1750 de nuestra era, que coincide con la definición del IPCC del inicio de la era industrial. Esta fecha de referencia se puede modificar (véase Antecedentes y Directrices), pero no podrá ser anterior al año 1500 ni posterior al año 1950 de nuestra era.

\section{b. Determinación del área de distribución adicional prevista}

El área de distribución adicional prevista incluye solo las áreas que: i) no forman parte del área de distribución autóctona; y ii) son actualmente adecuadas o es muy probable que lleguen a serlo en los próximos 100 años; y iii) es muy probable que lleguen a ser ocupadas por la especie en los próximos 100 años, con o sin ayuda humana (siempre que las que cuenten con ayuda humana se ajusten al Comité de Estándares y Peticiones de la UICN (2019)). Esta probabilidad puede evaluarse sobre la base de la modelización (por ejemplo, de los desplazamientos inducidos por el clima), las translocaciones previstas o la observación empírica de los cambios del área de distribución.

Debido a los horizontes temporales que se suelen utilizar para proyectar los cambios en el área de distribución provocados por el cambio climático, el área de distribución adicional prevista se utilizará normalmente solo para el futuro a largo plazo (es decir, para calcular el Potencial a largo plazo). A menos que algunas unidades espaciales dentro del área de distribución adicional prevista puedan ser ocupadas, o se conviertan en el centro de los esfuerzos de conservación, en los próximos 10 años, los escenarios futuros a corto plazo (para calcular la Dependencia de la conservación y la Ganancia de conservación) no tendrán que considerar el área de distribución adicional prevista.

\section{c. Individuos gestionados e introducidos}

Tanto en el caso del área de distribución autóctona como del área de distribución adicional prevista, al determinar el estado de la especie en cada unidad espacial, solo se deberán considerar los individuos "silvestres" de la especie. Para consultar la definición de "silvestre", véase la versión actual de las Directrices de la Lista Roja (Comité de Estándares y Peticiones de la UICN, 2019), sección 2.1.4.

Tanto en el caso del área de distribución autóctona como del área de distribución adicional prevista, las subpoblaciones silvestres resultantes de las introducciones fuera del área de distribución autóctona de la especie se deberán tener en cuenta a la hora de determinar el estado, siempre que se cumplan ciertas condiciones (véase la versión actual de las Directrices de la Lista Roja (UICN, 2019), sección 2.1.3). 


\section{Delineating spatial units}

Las unidades espaciales se utilizan para incorporar la representación, uno de los tres requisitos de una especie perteneciente a la categoría Totalmente recuperada (véase II.3). Una especie Totalmente recuperada se presenta como un conjunto de poblaciones funcionales en un conjunto representativo de ecosistemas y comunidades de su área de distribución. Una forma práctica de evaluar esta condición consiste en determinar el estado de la especie en cada una de las diversas unidades espaciales que componen su área de distribución, delimitadas para representar la variación de las condiciones o entornos ecológicos dentro del área de distribución de la especie. Las unidades espaciales deben elegirse cuidadosamente porque la Puntuación Verde tiene en cuenta el número de unidades. Dado que las unidades espaciales se valoran por igual en el cálculo de las puntuaciones verdes, se deberán definir de forma que representen áreas de importancia similar para la conservación de la especie, tanto en lo que respecta a la representación general como a las acciones de conservación, en toda la extensión del área de distribución autóctona y del área de distribución adicional prevista de la especie.

Las unidades espaciales pueden delimitarse según la subpoblación, las características ecológicas y geográficas y la ubicación, o una combinación de ellas. Son preferibles las subdivisiones específicas de las especies basadas en su biología, como las subpoblaciones (definidas en el Comité de Estándares y Peticiones de la UICN (2019)). Las subespecies, poblaciones, unidades genéticas, vías migratorias, unidades evolutivamente significativas y segmentos de población específicos están todos relacionados desde el punto de vista conceptual con la definición de subpoblación de la UICN. Aunque no son específicas de las especies, las divisiones basadas en las ecorregiones, los tipos de hábitat o los tipos de ecosistemas también se pueden utilizar para definir las unidades espaciales, ya que se definen en función de criterios ecológicos y, por tanto, captan los diferentes entornos ecológicos en los que existe o existía una especie. Las características geográficas (por ejemplo, cuencas hidrográficas, islas, lagos, cordilleras) pueden ser representativas de las subpoblaciones. La reciente fragmentación de las especies en "subpoblaciones" como resultado de la actividad humana no constituye una base válida para delimitar unidades espaciales, en el caso de que estas "subpoblaciones" estuvieran históricamente conectadas. Por último, las áreas que se definen por su vulnerabilidad respecto a un proceso de amenaza específico ("localidades" en UICN (2019)) pueden utilizarse para definir unidades espaciales, partiendo de la base de que el estado de la especie será similar en toda un área que sea objeto de una amenaza similar.

Muchas especies con un área de distribución restringida pueden evaluarse a partir de una sola unidad espacial, o de dos unidades espaciales (por ejemplo, una para el área de distribución de una especie existente y otra para el área de distribución de una especie extirpada). Este también puede ser el caso de una especie que siempre haya existido en un tipo de ecosistema muy específico o de una especie cuya función sea similar en los diferentes entornos ecológicos en los que existe. Respecto a otras especies, pueden ser necesarias tres o más unidades espaciales para representar la variedad de condiciones y contextos ecológicos en los que esa especie está o ha estado presente.

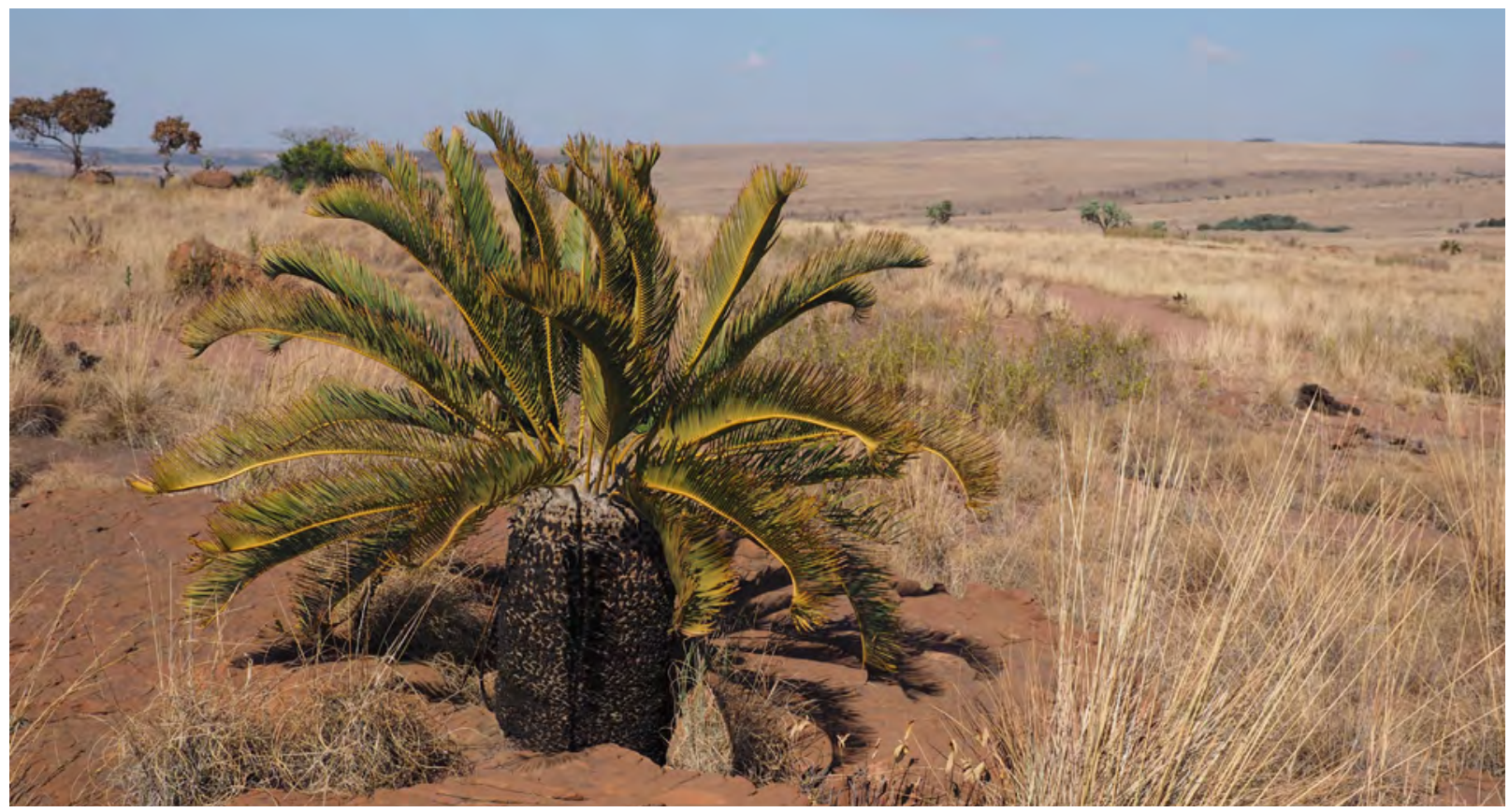

Cícadas del río Olifants (Encephalartos lanatus) @ C Michael Calonje 


\section{Evaluación del estado en una unidad espacial}

El estado de las especies en cada unidad espacial se evalúa de forma secuencial en el orden Ausente, Presente, Viable y Funcional. Para definir una especie como Ausente, Presente y Viable se debe recurrir a las categorías de la Lista Roja; por lo tanto, los evaluadores deben consultar las últimas versiones de los documentos de la Lista Roja (UICN, 2012a; UICN, 2012b; Comité de Estándares y Peticiones de la UICN, 2019). Los evaluadores deben documentar el estado más probable en cada unidad espacial, así como los estados plausibles mínimos y máximos, para reflejar el nivel de incertidumbre. A menos que exista una sola unidad espacial para toda la especie, se deben utilizar las orientaciones relativas a la inclusión en las listas rojas regionales.

\section{a. Evaluación de la ausencia}

La especie se considera Ausente en una unidad espacial si su categoría de la Lista Roja en la unidad espacial es Extinto (EX), Extinto en Estado Silvestre (EW), Extinto a Nivel Regional (RE), En Peligro Crítico (Posiblemente Extinto) (CR(PE)) o En Peligro Crítico (Posiblemente Extinto en Estado Silvestre) (CR(PEW)). Para obtener información sobre los métodos que se utilizan para determinar la categoría de Extinto frente a las etiquetas de Posiblemente Extinto, consulte la versión actual de las Directrices de la Lista Roja. La ponderación correspondiente a Ausente en la ecuación 1 es 0.

\section{b. Evaluación de la presencia}

La especie está Presente en una unidad espacial si tiene presencia (es decir, no está Ausente) y, o bien i) su categoría de la Lista Roja regional en la unidad espacial indica que está amenazada, es decir, Vulnerable (VU), En Peligro (EN) o En Peligro Crítico (CR), pero no CR(PE) o CR(PEW), o bien ii) la categoría es Casi Amenazado (NT) y la población de la unidad espacial está sufriendo una "disminución continua”, según la definición del Comité de Estándares y Peticiones de la UICN (2019). En las unidades espaciales que carecen de individuos maduros, la presencia de individuos inmaduros (como los bancos de semillas) califican la unidad espacial como Presente. Del mismo modo, las unidades espaciales con deuda de extinción (es decir, la falta de reclutamiento causará una posible extinción local) se evalúan como Presentes, no Ausentes. En una evaluación de la Lista Roja regional (UICN, 2012b) se debe prever la posibilidad de que se produzca un efecto de rescate como resultado de la inmigración desde otras unidades espaciales, lo que puede dar lugar a que disminuya la categoría de amenaza, por ejemplo, de VU a NT (véase UICN (2012b)). El estado de la especie en la unidad espacial debe basarse en la categoría después de que se haya realizado cualquier ajuste aplicable a la categoría de amenaza.

La ponderación por defecto para Presente en la ecuación 1 es 3. Los evaluadores pueden optar por asignar la ponderación correspondiente a la categoría específica de la Lista Roja regional para la población de la unidad espacial: 1,5 para CR, 2,5 para EN, 3,5 para VU, o 4,5 para NT con disminución continua (para ver una explicación de estas ponderaciones de alta resolución, véase Antecedentes y Directrices). Si la categoría de la Lista Roja es DD, utilice las ponderaciones de Ausente (cero) para el valor mínimo y Funcional (9) para el valor máximo.

\section{c. Evaluación de la viabilidad}

Una especie se considera Viable en una unidad espacial si una evaluación de la Lista Roja de la UICN regional (UICN, 2012b) de la especie en esa unidad espacial da como resultado una designación de Preocupación Menor (LC); o una designación de Casi Amenazada (NT) y la población de la unidad espacial no está sufriendo una "disminución continua" en su tamaño, según se define en el Comité de Estándares y Peticiones de la UICN (2019). Para realizar una evaluación de la Lista Roja regional (UICN, 2012b) se debe prever la posibilidad de que se produzca un efecto de rescate como resultado de la inmigración desde otras unidades espaciales, lo que puede dar lugar a que disminuya la categoría de amenaza, por ejemplo, de NT a LC (véase UICN (2012b)). El estado de la especie en la unidad espacial debe basarse en la categoría después de que se haya realizado cualquier ajuste aplicable a la categoría de amenaza.

La ponderación por defecto para Viable en la ecuación 1 es 6 . Los evaluadores pueden optar por asignar la ponderación de la siguiente lista que corresponda a la categoría específica de la Lista roja regional en la unidad espacial: 5,5 para NT sin disminución continua, o 6,5 para LC. Si la categoría es DD, utilice las ponderaciones de Ausente para el valor mínimo y Funcional para el valor máximo. 


\section{d. Evaluación de la funcionalidad}

La funcionalidad, al igual que la viabilidad, se evalúa dentro de cada unidad espacial. Para ello es necesario evaluar cada unidad espacial en relación con las funciones que comprenden los roles más importantes de la especie. Aunque es posible que no sea fácil determinar estas funciones, la incorporación de la funcionalidad siempre que sea posible constituye un elemento fundamental de una visión de conservación a la que aspirar. Sin embargo, la consideración de la funcionalidad en el contexto de la recuperación de las especies no debe interpretarse erróneamente como la priorización de la conservación de las especies en función de su importancia funcional.

A diferencia de los otros estados (Ausente, Presente y Viable), el Funcional no se basa en los criterios de la Lista Roja (sin embargo, véase la sección sobre la relación entre Viabilidad y Funcionalidad en Antecedentes y Directrices). La funcionalidad se puede determinar directamente, considerando las interacciones de la especie y sus contribuciones a los procesos del ecosistema; o indirectamente, buscando síntomas de funcionalidad reducida, de forma análoga al enfoque de la Lista Roja de identificar síntomas de viabilidad reducida (Akçakaya et al., 2020). Cuando no se puede identificar una función en una especie, se pueden utilizar varios indicadores indirectos para evaluar la funcionalidad, como la densidad de población o la estructura por edades en zonas de bajo impacto humano o conforme a una referencia histórica.

Aunque, en principio, una población puede contribuir a un proceso de los ecosistemas concreto incluso cuando se encuentre en alto riesgo de extirpación, a efectos del proceso de evaluación del Estado Verde, la puntuación correspondiente a Funcional solo se aplica a las unidades espaciales que también son Viables. Como excepción está el caso poco frecuente en que una unidad espacial con una población naturalmente pequeña ( $<1000$ individuos maduros) esté desempeñando sus funciones ecológicas en los niveles de referencia, pero sin cumplir los criterios para ser Viable porque sería evaluada como VU según el criterio D de la Lista Roja. Si la población de dicha unidad espacial no está disminuyendo, no se encuentra bajo una amenaza específica y no cumple otros criterios para considerarse como VU, se podrá evaluar como Funcional.

Si las unidades espaciales se basan en subpoblaciones, entonces una unidad espacial se puntuará como Funcional si la subpoblación es Funcional según la definición anterior. Si las unidades espaciales no se basan en subpoblaciones y hay múltiples subpoblaciones por unidad espacial, una unidad espacial se considerará Funcional si más de la mitad de sus subpoblaciones son Funcionales (sin embargo, si se utilizan ponderaciones de escala más reducida, el umbral será diferente; véase más adelante).

La ponderación por defecto para Funcional en la ecuación 1 es 9. Los evaluadores pueden optar por asignar la ponderación de la siguiente lista que corresponda a la proporción de subpoblaciones dentro de la unidad espacial que fueron evaluadas como Funcionales: 8 para $<40 \%$, 9 para $40-70 \%, 10$ para $>70 \%$.

\section{Elaboración del escenario Actual contrafactual}

El escenario Actual contrafactual es un presente alternativo, previsto para determinar cuál habría sido la Puntuación Verde Actual si no se hubiera llevado a cabo ninguna acción de conservación en el pasado. Para elaborar el escenario contrafactual es necesario determinar el modo en que el conjunto de todas las acciones de conservación desde 1950 ha afectado a la trayectoria de la población de la especie a lo largo de este período de tiempo, a fin de poder estimar cuál habría sido el estado hoy en día en cada una de las unidades espaciales si esas acciones de conservación no hubieran tenido lugar. El pensamiento contrafactual es una herramienta común en el ámbito de la conservación (véase Antecedentes y Directrices para obtener más detalles e instrucciones)

Al elaborar el escenario contrafactual, los tipos de información que deben tenerse en cuenta incluyen el tamaño y las tendencias de la población, los cambios en la distribución y la disponibilidad del hábitat, la gravedad, el alcance y la intensidad de las amenazas, así como todas las acciones de conservación que se han puesto en marcha desde el inicio de las principales acciones de conservación. Se deben tener en cuenta las acciones de conservación que estaban en marcha en 1950 y todas las que las sucedieron. Los evaluadores deberán indicar el año de inicio de las acciones de conservación pasadas que se hayan tenido en cuenta en su evaluación. Además, deberán considerar la información sobre la eficacia de cada tipo de acción de conservación dentro del contexto más amplio de los cambios en las presiones y las oportunidades de conservación respecto a la especie que se encuentra en la unidad espacial.

Si no se ha llevado a cabo ninguna acción de conservación en el pasado, el escenario actual contrafactual será el mismo que el actual. 


\section{Elaboración de escenarios futuros}

Un escenario futuro proyecta el estado futuro en cada unidad espacial bajo diferentes supuestos, con el propósito de calcular los indicadores de Ganancia de conservación, Dependencia de la conservación y Potencial de recuperación. Los tipos de información que se deben tener en cuenta en la elaboración de los escenarios futuros son similares a los del escenario actual contrafactual anteriormente mencionados. El evaluador deberá tener en cuenta tanto las amenazas actuales como las amenazas futuras plausibles. Las amenazas futuras deben basarse en pruebas concretas (como planes de desarrollo, proyecciones socioeconómicas, etc.) y no deben ser de carácter especulativo.

\section{a. Futuro con conservación}

Con respecto al escenario Futuro con conservación (para evaluar la Ganancia de conservación), los evaluadores deben considerar además los efectos probables de todas las intervenciones de conservación que se estén llevando a cabo actualmente o que están planificadas durante el período de evaluación de 10 años. Sin embargo, los evaluadores no deberán tener en cuenta las acciones de conservación que se contemplan, pero no se planifican (por ejemplo, cuando no se especifican objetivos claros, estimaciones de costos o plazos), o las acciones de conservación que se planifican pero que no se espera que se inicien dentro del plazo de 10 años. En cuanto a las acciones planificadas, los evaluadores deben formular suposiciones realistas sobre i) la probabilidad de que la acción se lleve a cabo, y ii) la probabilidad de que las acciones de conservación tengan un efecto positivo en la población de una especie en una unidad espacial determinada. En el caso de las acciones en curso, los evaluadores deben considerar la probabilidad ii). Los beneficios probables que se esperan de estas medidas de conservación deberían depender de estas probabilidades.

\section{b. Futuro sin conservación}

Con respecto al escenario Futuro sin conservación (para evaluar la Dependencia de la conservación), los evaluadores deben considerar los efectos probables de todas las intervenciones de conservación que se están llevando a cabo actualmente o que están planificadas, y luego eliminar sus efectos de las proyecciones, de forma similar a cuando se eliminan los efectos de las acciones de conservación pasadas en un escenario contrafactual.

\section{c. Referencia actual}

Los dos escenarios futuros descritos en las secciones anteriores (5a y 5b) se comparan con el escenario Referencia actual para calcular los indicadores de Ganancia de conservación y Dependencia de la conservación. La Referencia actual evalúa el estado probable de la población de la unidad espacial al cabo de 10 años, basándose únicamente en las acciones de conservación en curso (incluyendo las acciones que es muy probable que se apliquen en el plazo de un año, con la financiación y los permisos en vigor), teniendo en cuenta tanto las amenazas actuales como las amenazas futuras plausibles. Las amenazas futuras deben basarse en pruebas concretas (como planes de desarrollo, proyecciones socioeconómicas, etc.) y no deben ser de carácter especulativo. El escenario Referencia actual tiene en cuenta la posibilidad de que la población de una unidad espacial siga disminuyendo o se recupere, independientemente de las acciones de conservación planificadas o de su retirada. Si los evaluadores no desean calcular una Referencia actual, entonces se toma por defecto el estado Actual.

\section{d. Potencial a largo plazo}

En cuanto al escenario Potencial a largo plazo (para evaluar el Potencial de recuperación), los evaluadores deben prever el esfuerzo de conservación plausible y las innovaciones que podrían surgir en los próximos 100 años. Esto incluye las acciones que podrían llevarse a cabo para eliminar las amenazas y las oportunidades para la restauración del hábitat y el aumento de la conectividad. Este escenario debe ser realista, teniendo en cuenta las limitaciones biológicas de la especie (por ejemplo, la duración generacional y la tasa máxima de aumento de la población) y su hábitat (por ejemplo, las tasas de regeneración). También debe ser realista en cuanto a los factores sociales, culturales y económicos (por ejemplo, las tendencias previstas en materia de urbanización); sin embargo, el potencial a largo plazo no debe verse limitado por las restricciones políticas o presupuestarias actuales. Este escenario se compara con el estado Actual (no con la Referencia actual) para evaluar el Potencial de recuperación. El Potencial a largo plazo no tiene por objeto constituir una predicción exacta, ya que sería imposible predecir con exactitud todos los cambios naturales, sociales, económicos y tecnológicos que se producirán en los próximos 100 años. Se trata más bien de una expectativa razonable de cuánto podría recuperarse la especie, teniendo en cuenta lo que se sabe hoy en día. 


\section{Incorporación de incertidumbres}

La incertidumbre sobre el estado de la especie (Ausente, Presente, Viable, Funcional) en cada unidad espacial y para cada escenario debe indicarse de forma explícita especificando: i) el estado más bajo plausible; ii) el estado más alto plausible, y iii) el estado más probable (mejor). Estas incertidumbres se propagan para calcular los valores mínimos y máximos de los cuatro indicadores de impacto de la conservación (véanse Antecedentes y Directrices y Ak冈akaya et al. (2018) apéndice S1).

\section{Documentación de las evaluaciones}

Con el fin de asegurar la plena justificación de las evaluaciones y posibilitar el análisis de sus datos, se requiere un conjunto de información de apoyo mínima. Estos datos facilitan la transparencia y la repetibilidad y permiten a los usuarios buscar y encontrar información fácilmente en el sitio web. En la publicación Antecedentes y Directrices relativos al Estado Verde de las Especies de la UICN se incluyen orientaciones sobre lo siguiente: i) información de apoyo requerida para todas las evaluaciones del Estado Verde; ii) información de apoyo requerida en condiciones específicas; y iii) información de apoyo recomendada. Tenga en cuenta que los Estándares de Documentación se actualizarán periódicamente.

\section{Comunicación de los resultados de la evaluación}

La forma de presentar los resultados de una evaluación depende de quién y cómo los utilice. Es posible que para determinados públicos solo sean relevantes uno o dos de los cuatro indicadores de impacto de la conservación, mientras que para otros se puedan presentar los cuatro. En la mayoría de los análisis en los que se incluyen múltiples especies, y a efectos de la mayoría de las investigaciones, se deberán utilizar los valores numéricos (porcentuales) de los cuatro indicadores de impacto de la conservación, así como sus límites de incertidumbre. Para otros fines, los resultados pueden comunicarse como una combinación de estos resultados numéricos y categorías, en consonancia con las categorías y los umbrales especificados anteriormente (véase "Categorías y umbrales", IV.3).

\section{Evaluaciones regionales (incluidas las nacionales)}

Es posible realizar evaluaciones del Estado Verde a escalas espaciales regionales, aunque para ello se deben tener muy en cuenta los aspectos de "área de distribución autóctona y adicional prevista" y "partes del área de distribución" de la definición correspondiente a Totalmente recuperada. Se recomienda encarecidamente que las evaluaciones regionales (incluidas las nacionales) se realicen solo después de que se hayan completado los dos primeros pasos de las evaluaciones mundiales: la determinación del área de distribución autóctona y adicional prevista, y la delimitación de las unidades espaciales.

En la medida de lo posible, el área de distribución de la especie objeto de una evaluación regional debe incluir una o más de las unidades espaciales de la evaluación mundial en su totalidad. En otras palabras, en las evaluaciones regionales o nacionales se debe evitar dividir una unidad espacial determinada y delimitada a efectos de una evaluación mundial. La inclusión de unidades espaciales completas (de la evaluación mundial) en las evaluaciones regionales permitirá combinar los resultados de dos o más evaluaciones regionales y, por tanto, facilitar el flujo de información de las evaluaciones regionales a las mundiales. 


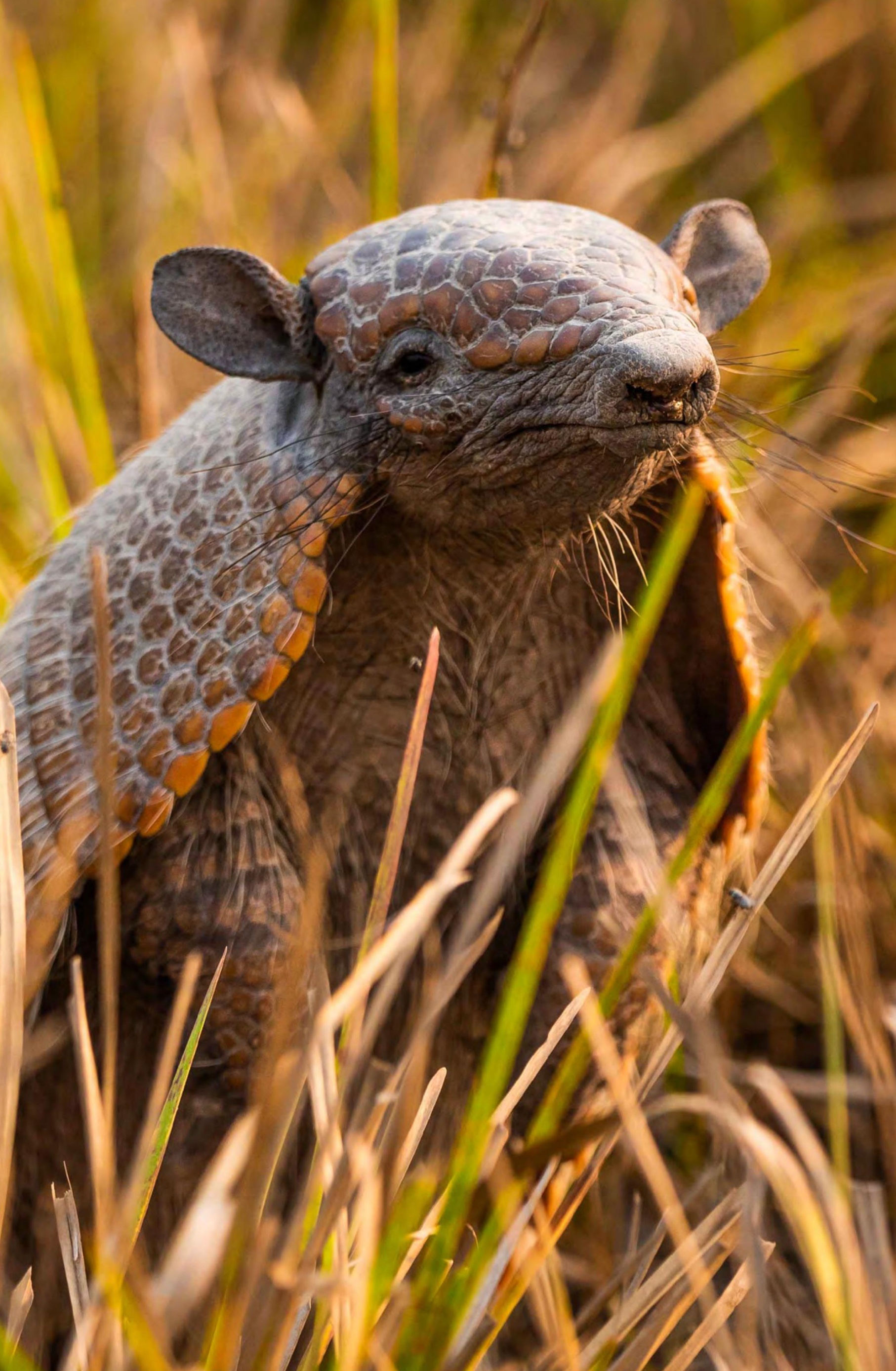




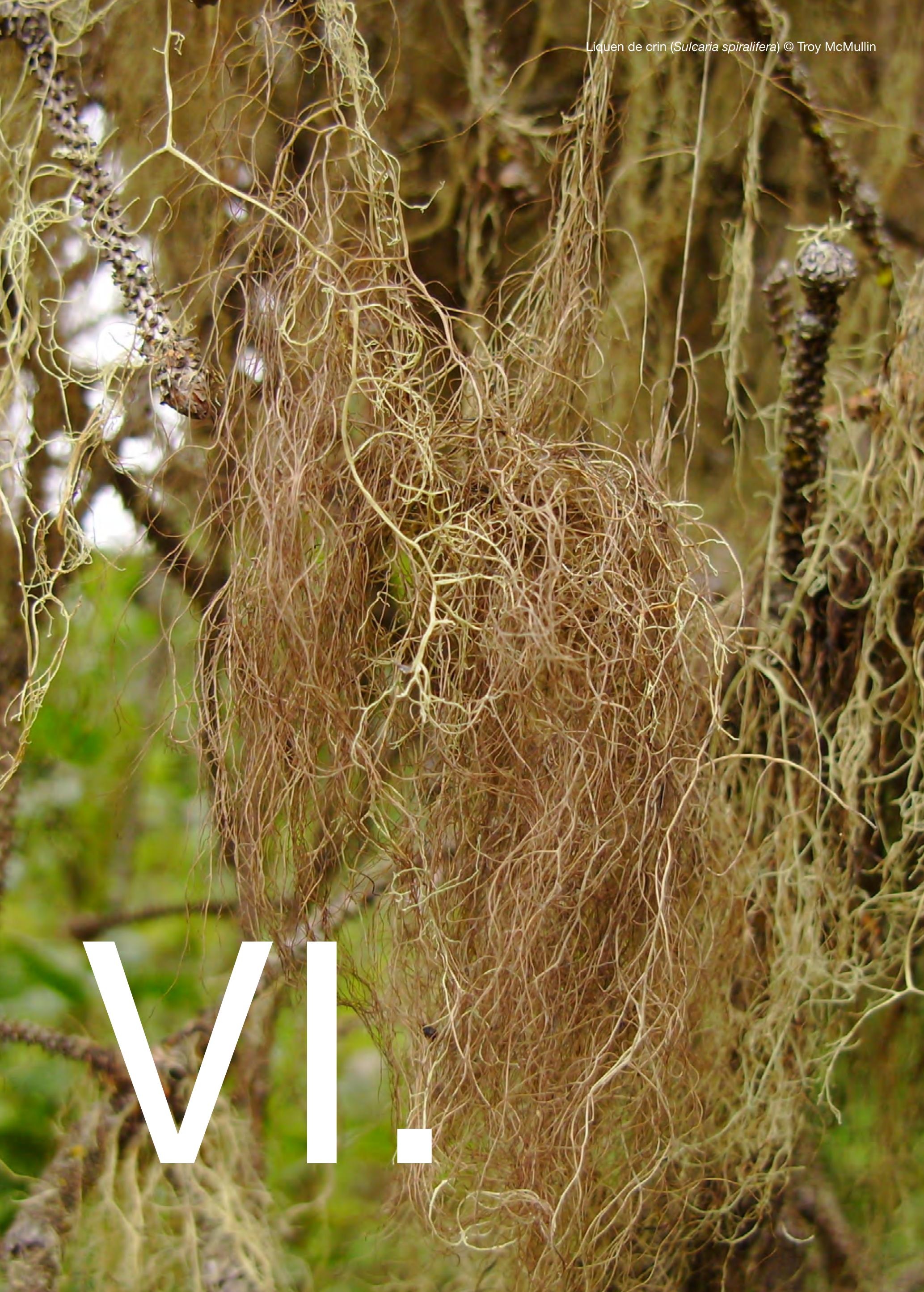




\section{Referencias}

Akçakaya, H.R., Bennett, E.L., Brooks, T.M., Grace, M.K., Heath, A., Hedges, S., Hilton-Taylor, C., Hoffmann, M., Keith, D.A., Long, B., Mallon, D.P., Meijaard, E., Milner-Gulland, E.J., Rodrigues, A.S.L., Rodriguez, J.P., Stephenson, P.J., Stuart, S.N. y Young, R.P. (2018). 'Quantifying species recovery and conservation success to develop an IUCN Green List of Species'. Conservation Biology 32:1128-1138. Disponible en el: https://doi.org/10.1111/cobi.13112 (fecha de consulta: 10 de setiembre de 2020).

Akçakaya, H.R., Rodrigues, A.S.L., Keith, D.A., Milner-Gulland, E.J., Sanderson, E.W., Hedges, S., Mallon, D.P., Grace, M.K., Long, B., Meijaard, E. y Stephenson, P.J. (2020). 'Assessing ecological function in the context of species recovery'. Conservation Biology 34:561-571. Disponible en el: https://doi.org/10.1111/cobi.13425 (fecha de consulta: 10 de setiembre de 2020).

Dudley, N. and Timmins, H.L. (eds.) (2021). A survey of user attitudes towards the proposed IUCN Green Status of Species. Gland, Switzerland: IUCN. https://doi.org/10.2305/IUCN.CH.2021.03.en

Comité de Estándares y Peticiones de la Unión Internacional para la Conservación de la Naturaleza (UICN) (2019). Directrices de uso de las Categorías y Criterios de la Lista Roja de la UICN. Versión 14. Publicación elaborada por el Subcomité de Estándares y Peticiones. Disponible en http://www.iucnredlist.org/documents/RedListGuidelines.pdf.

Comisión de Supervivencia de Especies (CSE) de la Unión Internacional para la Conservación de la Naturaleza (UICN) (2014). Directrices para reintroducciones y otras translocaciones para fines de conservación: Versión 1.0. Gland, Suiza: Comisión de Supervivencia de Especies de la UICN. https://portals.iucn.org/library/node/45234

International Union for Conservation of Nature (IUCN) and World Commission on Protected Areas (WCPA) (2017). IUCN Green List of Protected and Conserved Areas: Standard, Version 1.1. Gland, Switzerland: IUCN.

Klein Goldewijk, K., Beusen, A., van Drecht, G. y de Vos, M. (2011). 'The HYDE 3.1 spatially explicit database of human-induced global land-use change over the past 12,000 years'. Global Ecology and Biogeography 20:73-86. Disponible en el: https://doi.org/10.1111/j.1466-8238.2010.00587.x (fecha de consulta: 10 de setiembre de 2020).

Sanderson, E.W. (2019). 'A full and authentic reckoning of species' ranges for conservation: response to Akçakaya et al. 2018'. Conservation Biology 33:1208-1210. Disponible en el: https://doi.org/10.1111/cobi.13399 (fecha de consulta: 10 de setiembre de 2020).

Stephenson, P.J., Grace, M.K., Akçakaya, H.R., Rodrigues, A.S.L., Long, B., Mallon, D.P., Meijaard, E., Rodriguez, J.P., Young, R.P., Brooks, T.M. y Hilton-Taylor, C. (2019). 'Defining the indigenous ranges of species to account for geographic and taxonomic variation in the history of human impacts: reply to Sanderson 2019'. Conservation Biology 33:12111213. Disponible en el: https://doi.org/10.1111/cobi.13400 (fecha de consulta: 10 de setiembre de 2020).

Unión Internacional para la Conservación de la Naturaleza (UICN) (2012a). Categorías y Criterios de la Lista Roja de la UICN: Versión 3.1. Segunda edición. Gland, Suiza y Cambridge, Reino Unido: UICN. https://portals.iucn.org/library/ node/10316

Unión Internacional para la Conservación de la Naturaleza (UICN) (2012b). Directrices para el uso de los Criterios de la Lista Roja de la UICN a nivel regional y nacional: Versión 4.0. Gland, Suiza y Cambridge, Reino Unido: UICN. https:// portals.iucn.org/library/node/10338

Unión Internacional para la Conservación de la Naturaleza (UICN) y Comisión Mundial de Áreas Protegidas (CMAP). (2017). Lista Verde de la UICN de Áreas Protegidas y Conservadas: Estándar, Versión 1.1. Gland, Suiza: UICN. 




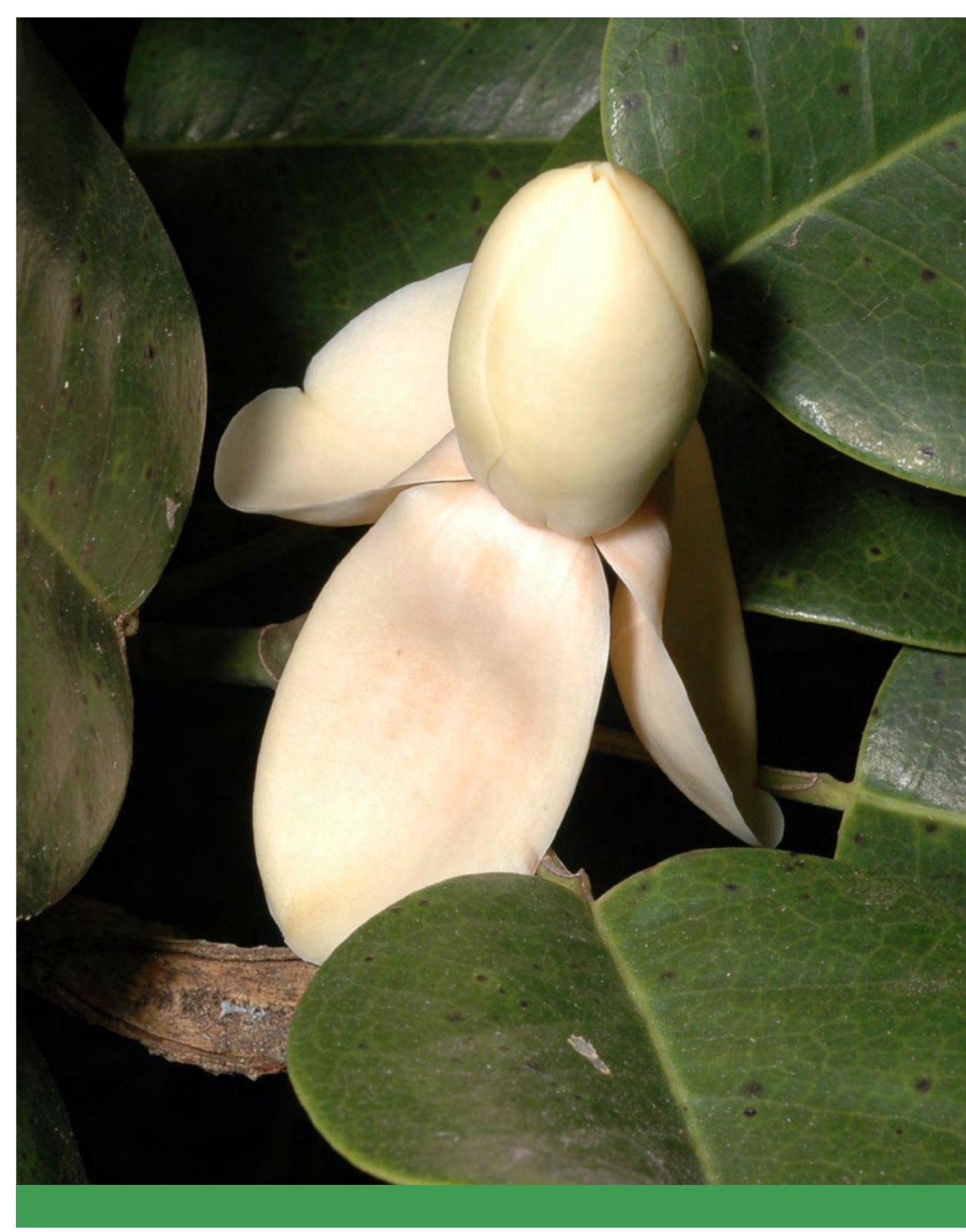

UNIÓN INTERNACIONAL PARA LA

CONSERVACIÓN DE LA NATURALEZA

SEDE MUNDIAL

Rue Mauverney 28

1196 Gland, Suiza

mail@iucn.org

Tel +41229990000

Fax +4122 9990002

www.iucn.org/es

www.iucn.org/resources/publications 